\title{
An estimate on the number of eigenvalues of a quasiperiodic Jacobi matrix of size $n$ contained in an interval of size $n^{-C}$
}

\author{
Ilia Binder and Mircea Voda
}

\begin{abstract}
We consider infinite quasi-periodic Jacobi self-adjoint matrices for which the three main diagonals are given via values of real analytic functions on the trajectory of the shift $x \rightarrow x+\omega$. We assume that the Lyapunov exponent $L\left(E_{0}\right)$ of the corresponding Jacobi cocycle satisfies $L\left(E_{0}\right) \geq \gamma>0$. In this setting we prove that the number of eigenvalues $E_{j}^{(n)}(x)$ of a submatrix of size $n$ contained in an interval $I$ centered at $E_{0}$ with $|I|=n^{-C_{1}}$ does not exceed $(\log n)^{C_{0}}$ for any $x$. Here $n \geq n_{0}$, and $n_{0}, C_{0}, C_{1}$ are constants depending on $\gamma$ (and the other parameters of the problem).
\end{abstract}

Mathematics Subject Classification (2010). Primary 81Q10; Secondary 47B36, 82B44.

Keywords. Eigenvalues, eigenfunctions, quasiperiodic Jacobi matrix, avalanche principle, large deviations.

\section{Contents}

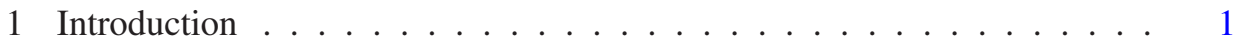

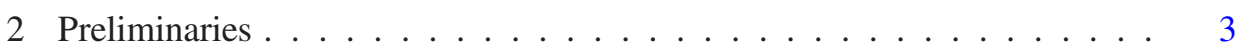

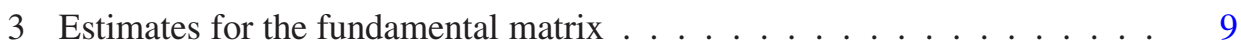

4 Estimates for the entries of the fundamental matrix . . . . . . . . 25

References . . . . . . . . . . . . . . . . . . . 45

\section{Introduction}

Denote $\mathbb{T} \stackrel{\text { def }}{=} \mathbb{R} / \mathbb{Z}$ and let $a: \mathbb{T} \rightarrow \mathbb{R}$, and $b: \mathbb{T} \rightarrow \mathbb{C}$ be real analytic functions, with $b$ not identically zero. Let $\omega \in(0,1)$ satisfy a (generic) Diophantine condition of the form

$$
\|n \omega\| \geq \frac{C_{\omega}}{n(\log n)^{\alpha}},
$$


where $\alpha>1$ is fixed. We consider the quasiperiodic Jacobi operator $H(x, \omega)$ defined on $l^{2}(\mathbb{Z})$ by

$$
\begin{aligned}
& {[H(x, \omega) \varphi](k)} \\
& \quad=-b(x+(k+1) \omega) \varphi(k+1)-\overline{b(x+k \omega)} \varphi(k-1)+a(x+k \omega) \varphi(k) .
\end{aligned}
$$

The important special case given by $b \equiv 1$ (Schrödinger operator) has been studied extensively (see the monograph [3]). The study of results that apply to quasiperiodic Jacobi operators in such a general setting has been launched by the recent work of Jitomirskaya, Koslover, and Schulteis [7] and Jitomirskaya and Marx [8]. In particular, they studied the extended Harper's model which corresponds to $a(x)=2 \cos (2 \pi x)$, $b(x)=\lambda_{1} e^{2 \pi i(x-\omega / 2)}+\lambda_{2}+\lambda_{3} e^{-2 \pi i(x-\omega / 2)}$ (see [6] and [9]). Further motivation for the study of these operators comes from the general fact that quasiperiodic Jacobi operators are necessary for the solution of the inverse spectral problem for discrete quasiperiodic operators of second order, and for the solution of the Toda lattice with quasiperiodic initial data.

The main objective of this work is to estimate the number of Dirichlet eigenvalues of the problem on a finite interval of length $n$ which fall into a given interval of size $n^{-C}$. This type of estimate plays a central role in the work of Goldstein and Schlag [4] and [5]. In our analysis we use many ideas and methods of their work. On the other hand, as it was noted in [8], the singularities (associated with the zeros of $b$ ) of the corresponding matrix-functions introduce considerable technical difficulties. These difficulties are addressed by using a large deviation theorem for subharmonic functions ([4], Theorem 3.8) applied to $\log |b|$, which will allow us to include the singularities in the exceptional sets. The derivation of the large deviation estimate for the characteristic polynomials via the method of [5] becomes especially complicated, even if $b$ would have no zeros. We show how to get around these difficulties by introducing a different derivation which makes a finer use of the cocycle structure (see the proof of Lemma 4.2). Our estimate on the number of eigenvalues also improves on the estimate in [5].

The methods we will employ are complex analytic, so from now on we canonically identify $\mathbb{T}$ with the unit circle in $\mathbb{C}$. It is known that $a$ and $b$ can be extended to be (complex) analytic on a neighborhood of $\mathbb{T}$. Let

$$
\tilde{b}(z) \stackrel{\text { def }}{=} \overline{b(1 / \bar{z})}
$$

denote the analytic extension of $\bar{b}$. We now extend the definition of $H(\cdot, \omega)$, to a neighborhood on which both $a$ and $b$ can be extended, by

$$
\begin{aligned}
& {[H(z, \omega) \varphi](k)} \\
& \quad=-b(z+(k+1) \omega) \varphi(k+1)-\tilde{b}(z+k \omega) \varphi(k-1)+a(z+k \omega) \varphi(k) .
\end{aligned}
$$

Note that $H(\cdot, \omega)$ is not necessarily self-adjoint off $\mathbb{T}$. We work with this extension because, for our methods to work, we want the determinant to be ana- 
lytic in the phase variable. For simplicity we make the notational convention that $z+k \omega \stackrel{\text { def }}{=} z \exp (2 \pi i k \omega)$, for $z \in \mathbb{C}$ and $k \in \mathbb{Z}$.

We consider the finite Jacobi submatrix on $[0, n-1]$, denoted by $H^{(n)}(z, \omega)$, and defined by

$$
\left[\begin{array}{ccccc}
a(z) & -b(z+\omega) & 0 & \cdots & 0 \\
-\tilde{b}(z+\omega) & a(z+\omega) & -b(z+2 \omega) & \ldots & 0 \\
\ddots & \ddots & \ddots & \ldots & \vdots \\
0 & \cdots & 0 & -\tilde{b}(z+(n-1) \omega) & a(z+(n-1) \omega)
\end{array}\right] .
$$

Let $L(E)$ be the Lyapunov exponent associated with $H(x, \omega)$; see (2.11). Our main result is as follows.

Main theorem. Assume that $E_{0} \in \mathbb{R}$ is such that $L\left(E_{0}\right) \geq \gamma>0$. Then there exist constants $C_{0}=C_{0}(\omega), C_{1}=C_{1}\left(a, b, E_{0}, \omega, \gamma\right)$, and $n_{0}=n_{0}\left(a, b, E_{0}, \omega, \gamma\right)$ such that for every $x \in \mathbb{T}$ and $n \geq n_{0}$ the number of eigenvalues for $H^{(n)}(x, \omega)$ located in $\left\{E:\left|E-E_{0}\right|<n^{-C_{1}}\right\}$ is at most $(\log n)^{C_{0}}$ and furthermore, for any $x_{0} \in \mathbb{T}$ and $n \geq n_{0}$ the number of zeros for $\operatorname{det}\left(H^{(n)}(\cdot, \omega)-E_{0}\right)$ contained in $\left\{z:\left|z-x_{0}\right|<n^{-1}\right\}$ is at most $(\log n)^{C_{0}}$.

In the Schrödinger case such estimates and further refinements were obtained by Goldstein and Schlag (see [5], Proposition 4.9). In fact we will prove a slightly stronger theorem, Theorem 4.13.

Acknowledgements. The authors are grateful to Michael Goldstein for suggesting the problem and for extensive discussions which were instrumental to the completion of the project. The first author was partially supported by the NSERC Discovery Grant 5810-2009-298433.

\section{Preliminaries}

We proceed by introducing some notation and giving an overview of the methods. For $\varphi$ satisfying the difference equation $H(z, \omega) \varphi=E \varphi$ let $M_{n}$ be the matrix such that

$$
\left[\begin{array}{c}
\varphi(n) \\
\varphi(n-1)
\end{array}\right]=M_{n}\left[\begin{array}{c}
\varphi(0) \\
\varphi(-1)
\end{array}\right], \quad n \geq 1
$$

We call $M_{n}$ the fundamental matrix. We clearly have

$$
M_{n}(z)=\prod_{j=n-1}^{0}\left(\frac{1}{b(z+(j+1) \omega)}\left[\begin{array}{cc}
a(z+j \omega)-E & -\tilde{b}(z+j \omega) \\
b(z+(j+1) \omega) & 0
\end{array}\right]\right),
$$


for $z$ such that $\prod_{j=1}^{n} b(z+j \omega) \neq 0$. Note that in order to simplify the notation we suppressed the dependence on $\omega$ and $E$. We will be doing this throughout the paper whenever possible. From now on, if needed, we will include the set on which the matrices $M_{n}$ are not defined in the exceptional sets.

It is straightforward to see that

$$
M_{n}(z)=\left[\begin{array}{cc}
f_{n}(z) & -\frac{\tilde{b}(z)}{b(z+\omega)} f_{n-1}(z+\omega) \\
f_{n-1}(z) & -\frac{\tilde{b}(z)}{b(z+\omega)} f_{n-2}(z+\omega)
\end{array}\right],
$$

with

$$
f_{n}(z)=\frac{1}{\prod_{j=1}^{n} b(z+j \omega)} f_{n}^{a}(z)
$$

where

$$
f_{n}^{a}(z)=\operatorname{det}\left[H^{(n)}(z, \omega)-E\right]
$$

(see [11], Chapter 1, where such relations are deduced in a detailed manner). Since $f_{n}^{a}(x, E)$ is the characteristic polynomial of $H^{(n)}(x, \omega)$ it is natural to estimate the number of eigenvalues by applying Jensen's formula to $f_{n}^{a}$. For this to work we need upper and lower estimates on $\log \left|f_{n}^{a}\right|$. These estimates will follow from the deviations estimates for the fundamental matrix and its entries (see Theorem 3.10 and Proposition 4.10).

The main tools for obtaining the deviations estimates for the fundamental matrix are a deviations estimate for subharmonic functions and the Avalanche Principle, both of which we recall next. In what follows $\mathcal{A}_{\rho}$ will denote the annulus $\{z \in \mathbb{C}:|z| \in$ $(1-\rho, 1+\rho)\}$ and we fix $p>\alpha+2$.

Theorem 2.1 ([4], Theorem 3.8). Let u be a subharmonic function and let

$$
u(z)=\int_{\mathbb{C}} \log |z-\zeta| d \mu(\zeta)+h(z)
$$

be its Riesz representation on a neighborhood of $\mathcal{A}_{\rho}$. If $\mu\left(\mathcal{A}_{\rho}\right)+\|h\|_{L^{\infty}\left(\mathcal{A}_{\rho}\right)} \leq M$, then for any $\delta>0$ and any positive integer $n$ we have

$$
\operatorname{mes}\left(\left\{x \in \mathbb{T}:\left|\sum_{k=1}^{n} u(x+k \omega)-n\langle u\rangle\right|>\delta n\right\}\right)<\exp \left(-c_{0} \delta n+r_{n}\right) \text {, }
$$

where $c_{0}=c_{0}(\omega, M, \rho)$ and

$$
r_{n}= \begin{cases}C_{0}(\log n)^{p}, & n>1 \\ C_{0}, & n=1\end{cases}
$$


with $C_{0}=C_{0}(\omega, p)$. If $p_{s} / q_{s}$ is a convergent of $\omega$ and $n=q_{s}>1$ then one can choose $r_{n}=C_{0} \log n$.

Proposition 2.2 (Avalanche Principle; [5], Proposition 3.3). Let $A_{1}, \ldots, A_{n}, n \geq 2$, be a sequence of $2 \times 2$ matrices. If

$$
\begin{gathered}
\max _{1 \leq j \leq n}\left|\operatorname{det} A_{j}\right| \leq 1, \\
\min _{1 \leq j \leq n}\left\|A_{j}\right\| \geq \mu>n,
\end{gathered}
$$

and

$$
\max _{1 \leq j<n}\left(\log \left\|A_{j+1}\right\|+\log \left\|A_{j}\right\|-\log \left\|A_{j+1} A_{j}\right\|\right)<\frac{1}{2} \log \mu
$$

then

$$
\left|\log \left\|A_{n} \ldots A_{1}\right\|+\sum_{j=2}^{n-1} \log \left\|A_{j}\right\|-\sum_{j=1}^{n-1} \log \left\|A_{j+1} A_{j}\right\|\right|<C_{0} \frac{n}{\mu}
$$

with some absolute constant $C_{0}$.

In [4] (where $b \equiv 1$ ) one takes advantage of the fact that $\log \left\|M_{n}(\cdot)\right\|$ is subharmonic (on a neighborhood of $\mathbb{T}$ ) and that it is almost invariant to get a first deviations estimate by using Theorem 2.1. Next, this estimate is used to apply the Avalanche Principle, which together with the almost invariance yields a sharper deviations estimate. Almost invariance refers to the fact that

$$
\left|\log \left\|M_{n}(x)\right\|-\frac{1}{l} \sum_{k=0}^{l-1} \log \left\|M_{n}(x+k \omega)\right\|\right| \leq C l, \quad x \in \mathbb{T} .
$$

In our case $\log \left\|M_{n}(\cdot)\right\|$ is not necessarily subharmonic, the Avalanche Principle (as stated) cannot be applied to $M_{n}$, because it possible that $\left|\operatorname{det} M_{n}\right| \not 1$, and the almost invariance may fail to hold on $\mathbb{T}$. To work around these issues it is natural to use the following two matrices associated with $M_{n}$ :

$$
M_{n}^{a}(z)=\left(\prod_{j=1}^{n} b(z+j \omega)\right) M_{n}(z)
$$

and

$$
M_{n}^{u}(z)=\frac{1}{\sqrt{\left|\operatorname{det} M_{n}(z)\right|}} M_{n}(z)=\left(\prod_{j=0}^{n-1}\left|\frac{b(z+(j+1) \omega)}{\tilde{b}(z+j \omega)}\right|^{1 / 2}\right) M_{n}(z)
$$

$M_{n}^{a}(\cdot)$ is analytic and hence $\log \left\|M_{n}^{a}(\cdot)\right\|$ is subharmonic, and $M_{n}^{u}(\cdot)$ is unimodular (i.e. $\left|\operatorname{det} M_{n}^{u}\right|=1$ ). Clearly, we will apply Theorem 2.1 to $\log \left\|M_{n}^{a}\right\|$ and the 
Avalanche Principle to $M_{n}^{u}$. Note that $\log \left\|M_{n}^{a}(\cdot)\right\|$ would be subharmonic even if we had $\bar{b}$ instead of $\tilde{b}$, however $\tilde{b}$ is needed to ensure that $f_{n}^{a}$ is analytic. Furthermore, if we have $\bar{b}$ instead of $\tilde{b}$ the function $\log \left|f_{n}^{a}(\cdot)\right|$ is not necessarily subharmonic. The use of such auxiliary matrices is common to all the existing work in the quasiperiodic Jacobi setting (see, for example, [7], [8], and [10]).

Using (2.1), (2.3), and (2.4) it is straightforward to check that

$$
M_{n}^{a}(z)=\left[\begin{array}{cc}
f_{n}^{a}(z) & -\tilde{b}(z) f_{n-1}^{a}(z+\omega) \\
b(z+n \omega) f_{n-1}^{a}(z) & -\tilde{b}(z) b(z+n \omega) f_{n-2}^{a}(z+\omega)
\end{array}\right]
$$

and

$$
\begin{aligned}
& M_{n}^{u}(z) \\
& =\left[\begin{array}{cr}
f_{n}^{u}(z) & -\frac{\tilde{b}(z)}{b(z+\omega)}\left|\frac{b(z+\omega)}{\tilde{b}(z)}\right|^{1 / 2} f_{n-1}^{u}(z+\omega) \\
\left|\frac{b(z+n \omega)}{\tilde{b}(z+(n-1) \omega)}\right|^{1 / 2} f_{n-1}^{u}(x) & -\frac{\tilde{b}(z)}{b(z+\omega)}\left|\frac{b(z+n \omega) b(z+\omega)}{\tilde{b}(z+(n-1) \omega) \tilde{b}(z)}\right|^{1 / 2} f_{n-2}^{u}(z+\omega)
\end{array}\right],
\end{aligned}
$$

where

$$
f_{n}^{u}(z)=\left(\prod_{j=0}^{n-1}\left|\frac{b(z+(j+1) \omega)}{\tilde{b}(z+j \omega)}\right|^{1 / 2}\right) f_{n}(z)
$$

( $f_{n}$ and $f_{n}^{a}$ have already been defined).

Let

$$
S_{n}(z)=\sum_{k=0}^{n-1} \log |b(z+k \omega)|
$$

and

$$
\tilde{S}_{n}(z)=\sum_{k=0}^{n-1} \log |\tilde{b}(z+k \omega)|
$$

From (2.3) and (2.4) we get

$$
\log \left\|M_{n}(z)\right\|=-S_{n}(z+\omega)+\log \left\|M_{n}^{a}(z)\right\|
$$

and

$$
\log \left\|M_{n}^{u}(z)\right\|=-\frac{1}{2}\left(\widetilde{S}_{n}(z)+S_{n}(z+\omega)\right)+\log \left\|M_{n}^{a}(z)\right\| .
$$

It will be easy to see that these relations together with Theorem 2.1 applied to $\log |b|$ and $\log |\tilde{b}|$ allow us to pass from deviations estimates for $M_{n}^{a}$ to deviations estimates for $M_{n}$ and $M_{n}^{u}$ (see for example Corollary 3.6). 
Even though we will apply the Avalanche Principle to $M_{n}^{u}$ the conclusion will also hold for $M_{n}^{a}$ and $M_{n}$. We will make this more precise. Let $n=\sum_{j=1}^{m} l_{j}$, $s_{k}=\sum_{j=1}^{k} l_{j}$ where $m, l_{1}, \ldots, l_{m}$ are positive integers. We assume that $s_{0}=0$. By saying that, for example, the conclusion of the Avalanche Principle applied to $M_{n}^{u}$ also holds for $M_{n}^{a}$ we mean that

$$
\begin{aligned}
& \log \left\|M_{n}^{u}(z)\right\|+\sum_{j=2}^{m-1} \log \left\|M_{l_{j}}^{u}\left(z+s_{j-1} \omega\right)\right\| \\
& \quad-\sum_{j=1}^{m-1} \log \left\|M_{l_{j+1}}^{u}\left(z+s_{j} \omega\right) M_{l_{j}}^{u}\left(z+s_{j-1} \omega\right)\right\| \\
& =\log \left\|M_{n}^{a}(z)\right\|+\sum_{j=2}^{m-1} \log \left\|M_{l_{j}}^{a}\left(z+s_{j-1} \omega\right)\right\| \\
& \quad-\sum_{j=1}^{m-1} \log \left\|M_{l_{j+1}}^{a}\left(z+s_{j} \omega\right) M_{l_{j}}^{a}\left(z+s_{j-1} \omega\right)\right\| .
\end{aligned}
$$

This follows easily from (2.9).

The deviations estimate for $\log \left|f_{n}^{a}\right|$ is just the John-Nirenberg inequality. The needed BMO norm bound will be obtained by using the "BMO splitting lemma" [1], Lemma 2.3. As in the case for the fundamental matrix, we first obtain a rough estimate (Lemma 4.9) that allows us to apply the Avalanche Principle in order to obtain a better estimate. We follow the approach from [5] with the notable exception of the proof of Lemma 4.2 (cf. [5], Lemma 2.7). This is the only place where the difficulties come not only from the possible zeroes of $b$ but also from the fact that $b$ is not constant.

We will obtain a uniform upper bound for $\log \left|f_{n}^{a}(\cdot)\right|$ on $\mathbb{T}$ from an uniform upper bound for $\log \left\|M_{n}^{a}(\cdot)\right\|$ (Proposition 3.14) and the obvious inequality $\log \left|f_{n}^{a}(\cdot)\right|$ $\leq \log \left\|M_{n}^{a}(\cdot)\right\|$. The proof of Proposition 3.14 requires that the deviations estimate for $\log \left\|M_{n}^{a}\right\|$ holds on $r \mathbb{T}$ for $r$ in a neighborhood of 1 . Of course this implies that all the results leading to the deviations estimate should also hold on $r \mathbb{T}$. For simplicity we will prove these estimates on $\mathbb{T}$, however the proofs will be such that the generalization from $\mathbb{T}$ to $r \mathbb{T}$ is immediate. To this end the derivations up to Proposition 3.14 won't use the fact that $\tilde{b}=\bar{b}$ on $\mathbb{T}$. However, after that point we only need the results to hold on $\mathbb{T}$ and we will make use of said fact to simplify notation.

The deviations estimates will rely on the positivity of the Lyapunov exponent. Let

$$
\begin{aligned}
& L_{n}(r)=\frac{1}{n} \int_{\mathbb{T}} \log \left\|M_{n}(r x)\right\| d x, \\
& L_{n}^{u}(r)=\frac{1}{n} \int_{\mathbb{T}} \log \left\|M_{n}^{u}(r x)\right\| d x,
\end{aligned}
$$




$$
\begin{aligned}
L_{n}^{a}(r) & =\frac{1}{n} \int_{\mathbb{T}} \log \left\|M_{n}^{a}(r x)\right\| d x, \\
D(r) & =\int_{\mathbb{T}} \log |b(r x)| d x,
\end{aligned}
$$

and

$$
\tilde{D}(r)=\int_{\mathbb{T}} \log |\tilde{b}(r x)| d x .
$$

When $r=1$ we will omit the $r$ argument, so for example we will write $L_{n}$ instead of $L_{n}(1)$. The quantity $D$ appears naturally whenever one has to deal with the singularities given by the zeros of $b$ (see, for example, [8] and [10]). The quantities $L_{n}^{a}(r), D(r)$, and $\widetilde{D}(r)$ are finite because the integrands are subharmonic (and not identically $-\infty)$, and $L_{n}(r)$ is finite because from (2.8) we have

$$
L_{n}(r)=-D(r)+L_{n}^{a}(r) .
$$

By Kingman's subadditive ergodic theorem the following limits exist:

$$
\begin{gathered}
L(r)=\lim _{n \rightarrow \infty} L_{n}(r)=\inf _{n \geq 1} L_{n}(r), \\
L^{u}(r)=\lim _{n \rightarrow \infty} L_{n}^{u}(r)=\inf _{n \geq 1} L_{n}^{u}(r),
\end{gathered}
$$

and

$$
L^{a}(r)=\lim _{n \rightarrow \infty} L_{n}^{a}(r)=\inf _{n \geq 1} L_{n}^{a}(r) .
$$

$L=L(E, \omega)$ is called the Lyapunov exponent. From (2.9) it can be seen that

$$
L^{u}(r)=\frac{1}{2}(\tilde{D}(r)-D(r))+L(r)
$$

and in particular, since $D=\tilde{D}$, we have $L=L^{u}$. Since $M_{n}^{u}$ is unimodular it follows that $L_{n}^{u}(r) \geq 0$, and hence $L^{u}(r) \geq 0$. In particular we have that $L=L^{u} \geq 0$.

Fix $\gamma>0$. From now on we assume that $L \geq \gamma>0$. This assumption is needed to apply the Avalanche Principle, so in fact we will use $L^{u}=L \geq \gamma>0$. For the results to hold on $r \mathbb{T}, r \neq 1$, we will need that $r$ is close enough to 1 so that $L^{u}(r) \geq \gamma / 2>0$. Note that the results up to Lemma 3.9 don't use the Avalanche Principle and so they hold without the assumption that $L \geq \gamma>0$.

Henceforth we will assume that $a$ and $b$ are analytic on the closure of $\mathcal{A}_{\rho_{0}^{\prime \prime}}$ with $\rho_{0}^{\prime \prime}>0$ fixed. We also fix $\rho_{0}$ and $\rho_{0}^{\prime}$ such that $0<\rho_{0}<\rho_{0}^{\prime}<\rho_{0}^{\prime \prime}$. The reason for this setup is that $\log \left\|M_{n}^{a}(\cdot)\right\|$ will have a Riesz representation on $\mathcal{A}_{\rho_{0}^{\prime}}$, but we will be able to get the estimates on the Riesz representation (needed for Theorem 2.1) only on $\mathcal{A}_{\rho_{0}}$. The estimates before Proposition 3.14 will hold on $r \mathbb{\mathbb { T }}$ for every $r \in$ $\left(1-\rho_{0} / 2,1+\rho_{0} / 2\right)$ (provided $\left.L^{u}(r)>0\right)$ and the constants can be chosen uniformly

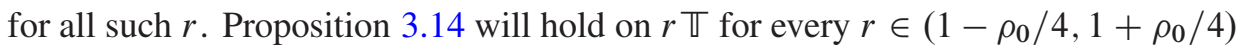
(provided $L^{u}(r)>0$ ). 


\section{Estimates for the fundamental matrix}

First we prove the almost invariance of $M_{n}^{a}$; see (3.7). The following lemma and its corollaries contain the main estimates that are needed to deal with the fact that $b$ could have zeros. If $b$ doesn't have any zeros then all the estimates hold trivially without exceptional sets and everything goes as in [4].

In what follows we will keep track of the dependence of the various constants on the parameters of our problem. The dependence on $\omega$ will only come up through Theorem 2.1. In order to simplify the notation we won't record the dependence on $\rho_{0}$, $\rho_{0}^{\prime}$, and $\rho_{0}^{\prime \prime}$ (except in the lemmas where $\rho_{0}$ appears in the statement). Dependence on any other quantities is such that if the quantity takes values in a compact set, then the constant can be chosen uniformly with respect to that quantity. The main dependence we are interested in, is that on $|E|$. We denote by $\|\cdot\|_{\infty}$ the $L^{\infty}$ norm on $\mathcal{A}_{\rho_{0}^{\prime \prime}}$ and we let

$$
\|b\|_{*}=\|b\|_{\infty}+\sup _{r \in\left(1-\rho_{0}, 1+\rho_{0}\right)}|D(r)| .
$$

Note that $\|b\|_{*}=\|\tilde{b}\|_{*}$. Also, note that, unless otherwise stated, the constants in different results are different.

Lemma 3.1. There exist constants

$$
\lambda_{0}=\lambda_{0}\left(\|a\|_{\infty},\|b\|_{*},|E|, \omega\right)
$$

and

$$
c_{0}=c_{0}\left(\|b\|_{*}, \omega\right)
$$

such that the following inequalities hold for any positive integer $l$ and any $\lambda \geq \lambda_{0}$ up to a set (independent of $E$ ) of measure less than $\exp \left(-c_{0} \lambda l\right)$ :

$$
\left|\log \left\|M_{l}^{a}(x)\right\|\right| \leq \lambda l
$$

and

$$
\left|\log \left\|M_{l}^{a}(x)^{-1}\right\|\right| \leq \lambda l
$$

Proof. There exists a constant $C=C\left(\|a\|_{\infty},\|b\|_{\infty},|E|\right)>0$ such that

$$
\log \left\|M_{l}^{a}(x)\right\| \leq C l
$$

for all $x$. On the other hand

$$
\left\|M_{l}^{a}(x)\right\| \geq\left|\operatorname{det} M_{l}^{a}(x)\right|^{1 / 2}=\prod_{j=0}^{l-1}|\tilde{b}(x+j \omega) b(x+(j+1) \omega)|^{1 / 2}
$$

for all $x$. Hence

$$
\frac{\widetilde{S}_{l}(x)+S_{l}(x+\omega)}{2} \leq \log \left\|M_{l}^{a}(x)\right\| \leq C l
$$


for all $x$. From Theorem 2.1 we can conclude that for any $\lambda^{\prime}>0$ we have

$$
-2 \lambda^{\prime} l \leq\left(\frac{\tilde{D}+D}{2}-\lambda^{\prime}\right) l \leq \log \left\|M_{l}^{a}(x)\right\| \leq C l \leq 2 \lambda^{\prime} l
$$

up to a set not exceeding $2 \exp \left(-c \lambda^{\prime} l+r_{l}\right)$ in measure, provided

$$
\lambda^{\prime} \geq \max \{-(\tilde{D}+D), C\} / 2 \text {. }
$$

By setting $\lambda=2 \lambda^{\prime}$ and choosing $\lambda_{0} \geq \max \{-(\widetilde{D}+D), C\}$ we have that (3.1) holds up to a set of measure not exceeding $2 \exp \left(-c \lambda l+r_{l}\right)$. Finally, it is easy to see that by choosing $\lambda_{0}$ such that

$$
\lambda_{0} \geq \frac{2}{c} \sup _{l \geq 1} \frac{\log 2+r_{l}}{l}
$$

we have

$$
2 \exp \left(-c \lambda l+r_{l}\right) \leq \exp \left(-\frac{c}{2} \lambda l\right), \lambda \geq \lambda_{0}, l \geq 1 .
$$

This concludes the proof of (3.1).

Since for almost every $x$ we have

$$
\begin{aligned}
& {\left[\begin{array}{cc}
a(x+j \omega)-E & -\tilde{b}(x+j \omega) \\
b(x+(j+1) \omega) & 0
\end{array}\right]^{-1}} \\
& \quad=\frac{1}{\tilde{b}(x+j \omega) b(x+(j+1) \omega)}\left[\begin{array}{ccc}
0 & \tilde{b}(x+j \omega) \\
-b(x+(j+1) \omega) & a(x+j \omega)-E
\end{array}\right]
\end{aligned}
$$

it is straightforward to see that there exists a constant $C=C\left(\|a\|_{\infty},\|b\|_{\infty},|E|\right)$ such that

$$
-\frac{\widetilde{S}_{l}(x)+S_{l}(x+\omega)}{2} \leq \log \left\|M_{l}^{a}(x)^{-1}\right\| \leq C l-\widetilde{S}_{l}(x)-S_{l}(x+\omega)
$$

for almost every $x$. Now (3.2) follows in the same way as (3.1). Note that the exceptional set comes from $\widetilde{S}_{l}(x)+S_{l}(x+\omega)$ and is thus independent of $E$.

The same type of estimates can be obtained now for $M_{n}$ and $M_{n}^{u}$. We just record one of the estimates that will be needed later.

Corollary 3.2. There exist constants

$$
\lambda_{0}=\lambda_{0}\left(\|a\|_{\infty},\|b\|_{*},|E|, \omega\right)
$$

and

$$
c_{0}=c_{0}\left(\|b\|_{*}, \omega\right)
$$

such that

$$
\left|\log \left\|M_{l}^{u}(x)^{-1}\right\|\right| \leq \lambda l
$$

holds for any positive integer $l$ and any $\lambda \geq \lambda_{0}$ up to a set of measure less than $\exp \left(-c_{0} \lambda l\right)$. 
Proof. From (2.4) we have

$$
\log \left\|M_{l}^{u}(x)^{-1}\right\|=\frac{1}{2}\left(\widetilde{S}_{l}(x)+S_{l}(x+\omega)\right)+\log \left\|M_{l}^{a}(x)^{-1}\right\| .
$$

Using Theorem 2.1 and (3.2) we get

$$
-3 \lambda^{\prime} l \leq\left(\frac{\tilde{D}+D}{2}-2 \lambda^{\prime}\right) l \leq \log \left\|M_{l}^{u}(x)^{-1}\right\| \leq\left(\frac{\widetilde{D}+D}{2}+2 \lambda^{\prime}\right) l \leq 3 \lambda^{\prime} l
$$

up to a set of measure less than $2 \exp \left(-c_{1} \lambda^{\prime} l+r_{l}\right)+\exp \left(-c_{2} \lambda^{\prime} l\right) \leq \exp \left(-c 3 \lambda^{\prime} l\right)$ provided $\lambda^{\prime}$ is large enough. Now we can take $\lambda=3 \lambda^{\prime}$.

Corollary 3.3. There exist constants

$$
\lambda_{0}=\lambda_{0}\left(\|a\|_{\infty},\|b\|_{*},|E|, \omega\right)
$$

and

$$
c_{0}=c_{0}\left(\|b\|_{*}, \omega\right)
$$

such that the following inequalities hold for any positive integers $l$ and $n$, and any $\lambda \geq \lambda_{0}$ up to a set (depending on $n$ ) of measure less than $\exp \left(-c_{0} \lambda l\right)$ :

$$
\begin{aligned}
\left|\log \left\|M_{l}^{a}(x)\right\|-l L_{l}^{a}\right| & \leq \lambda l, \\
\left|\log \left\|M_{n+l}^{a}(x)\right\|-\log \left\|M_{n}^{a}(x)\right\|\right| & \leq \lambda l, \\
\left|\log \left\|M_{n}^{a}(x+l \omega)\right\|-\log \left\|M_{n}^{a}(x)\right\|\right| & \leq \lambda l,
\end{aligned}
$$

and

$$
\left|\log \left\|M_{n}^{a}(x)\right\|-\frac{1}{l} \sum_{k=0}^{l-1} \log \left\|M_{n}^{a}(x+k \omega)\right\|\right| \leq \lambda l .
$$

Proof. By integrating (3.3) we get

$$
\frac{\widetilde{D}+D}{2} \leq L_{l}^{a} \leq C
$$

This and (3.1) imply (3.4).

We have

$$
M_{n+l}^{a}(x)=M_{l}^{a}(x+n \omega) M_{n}^{a}(x),
$$

hence

$$
-\log \left\|M_{l}^{a}(x+n \omega)^{-1}\right\| \leq \log \left\|M_{n+l}^{a}(x)\right\|-\log \left\|M_{n}^{a}(x)\right\| \leq \log \left\|M_{l}^{a}(x+n \omega)\right\|
$$

for almost every $x$. Now (3.5) follows by (3.1) and (3.2). 
From the fact that

$$
M_{n}^{a}(x+l \omega) M_{l}^{a}(x)=M_{l}^{a}(x+n \omega) M_{n}^{a}(x)
$$

we conclude that

$$
\begin{aligned}
-\log & \left\|M_{l}^{a}(x+n \omega)^{-1}\right\|-\log \left\|M_{l}^{a}(x)\right\| \\
& \leq \log \left\|M_{n}^{a}(x+l \omega)\right\|-\log \left\|M_{n}^{a}(x)\right\| \\
& \leq \log \left\|M_{l}^{a}(x+n \omega)\right\|+\log \left\|M_{l}^{a}(x)^{-1}\right\|
\end{aligned}
$$

for almost every $x$. Now (3.6) also follows by (3.1) and (3.2).

Let $\lambda \geq \lambda_{0}$. Then for $k=1, \ldots, l-1$ we have $\lambda l / k>\lambda_{0}$, so by (3.6) we get

$$
\left|\log \left\|M_{n}^{a}(x+k \omega)\right\|-\log \left\|M_{n}^{a}(x)\right\|\right| \leq\left(\frac{\lambda l}{k}\right) k=\lambda l
$$

up to a set of measure less than $\exp (-c \lambda l)$. Summing over $k=0, \ldots, l-1$ and dividing by $l$ we get that (3.7) holds up to a set of measure less than $l \exp (-c \lambda l)$. Finally, note that $l \exp (-c \lambda l)<\exp (-c \lambda l / 2), l \geq 1$ if $\lambda$ is large enough. This concludes the proof.

Next we provide bounds on the Riesz representation of $\log \left\|M_{n}^{a}(\cdot)\right\|$, which are needed to ensure that the constants we will get from Theorem 2.1 don't depend on $n$.

Lemma 3.4. Let

$$
\frac{1}{n} \log \left\|M_{n}^{a}(z)\right\|=\int_{\mathcal{A}_{\rho_{0}^{\prime}}} \log |z-\zeta| d \mu_{n}(\zeta)+h_{n}(z)
$$

be the Riesz representation on $\mathcal{A}_{\rho_{0}^{\prime}}$. There exists a constant

$$
C_{0}=C_{0}\left(\|a\|_{\infty},\|b\|_{*},|E|, \rho_{0}, \rho_{0}^{\prime}, \rho_{0}^{\prime \prime}\right)
$$

such that

$$
\mu_{n}\left(\mathcal{A}_{\rho_{0}}\right)+\left\|h_{n}\right\|_{L^{\infty}\left(\mathcal{A}_{\rho_{0}}\right)} \leq C_{0} .
$$

Proof. Let $u_{n}(z)=\log \left\|M_{n}^{a}(z)\right\| / n$ and $T_{n}=\sup _{\mathcal{A}_{\rho_{0}^{\prime \prime}}} u_{n}$. From [5], Lemma 2.2, we have that

$$
\begin{aligned}
\mu_{n}\left(\mathcal{A}_{\rho_{0}}\right) & \leq \mu_{n}\left(\mathcal{A}_{\rho_{0}^{\prime}}\right) \\
& \leq C\left(\rho_{0}^{\prime}, \rho_{0}^{\prime \prime}\right)\left(T_{n}-\sup _{\mathcal{A}_{\rho_{0}^{\prime}}} u_{n}\right) \\
& \leq C\left(T_{n}-\sup _{\mathbb{T}} u_{n}\right) \\
& \leq C\left(T_{n}-L_{n}^{a}\right)
\end{aligned}
$$


and

$$
\begin{aligned}
\left\|h_{n}\right\|_{L^{\infty}\left(\mathcal{A}_{\rho_{0}}\right)} & \leq\left\|h_{n}-\sup _{\mathcal{A}_{\rho_{0}^{\prime}}} u_{n}\right\|_{L^{\infty}\left(\mathcal{A}_{\rho_{0}}\right)}+\sup _{\mathcal{A}_{\rho_{0}^{\prime}}} u_{n} \\
& \leq C\left(\rho_{0}, \rho_{0}^{\prime}, \rho_{0}^{\prime \prime}\right)\left(T_{n}-\sup _{\mathcal{A}_{\rho_{0}^{\prime}}} u_{n}\right)+T_{n} \\
& \leq C\left(T_{n}-L_{n}^{a}\right)+T_{n} .
\end{aligned}
$$

The conclusion now follows from the fact that there exists a constant

$$
C=C\left(\|a\|_{\infty},\|b\|_{\infty},|E|, \rho_{0}^{\prime \prime}\right)
$$

such that $T_{n} \leq C$, and from (3.8).

Now we can prove the first deviations estimate.

Lemma 3.5. Let $\delta_{0}>0$. For any $\delta \in\left(0, \delta_{0}\right)$ and any integer $n>1$ we have

$$
\operatorname{mes}\left\{x \in \mathbb{T}:\left|\log \left\|M_{n}^{a}(x)\right\|-n L_{n}^{a}\right|>n \delta\right\}<\exp \left(-c_{0} n \delta^{2}+C_{0}(\log n)^{p}\right),
$$

where

$$
c_{0}=c_{0}\left(\|a\|_{\infty},\|b\|_{*},|E|, \omega, \delta_{0}\right)
$$

and

$$
C_{0}=C_{0}\left(\|a\|_{\infty},\|b\|_{*},|E|, \omega, p, \delta_{0}\right)
$$

Proof. We have

$$
\begin{aligned}
& \operatorname{mes}\left\{x \in \mathbb{T}:\left|\log \left\|M_{n}^{a}(x)\right\|-n L_{n}^{a}\right|>n \delta\right\} \\
& \leq \operatorname{mes}\left\{x \in \mathbb{T}:\left|\frac{1}{n} \log \left\|M_{n}^{a}(x)\right\|-\frac{1}{l} \sum_{k=0}^{l-1} \frac{1}{n} \log \left\|M_{n}^{a}(x+k \omega)\right\|\right|>\frac{\delta}{2}\right\} \\
& \quad+\operatorname{mes}\left\{x \in \mathbb{T}:\left|\frac{1}{l} \sum_{k=0}^{l-1} \frac{1}{n} \log \left\|M_{n}^{a}(x+k \omega)\right\|-L_{n}^{a}\right|>\frac{\delta}{2}\right\} .
\end{aligned}
$$

The conclusion will follow by estimating the two quantities on the right-hand side of the above inequality.

From (3.7) we get

$$
\left|\log \left\|M_{n}^{a}(x)\right\|-\frac{1}{l} \sum_{k=0}^{l-1} \log \left\|M_{n}^{a}(x+k \omega)\right\|\right| \leq C_{1} l
$$


up to a set not exceeding $\exp (-c l)$ in measure. Let $l=\left[\delta n / 2 C_{1}\right]+1$. We have

$$
\frac{\delta}{2}<\frac{C_{1} l}{n}
$$

so we get

$$
\left|\frac{1}{n} \log \left\|M_{n}^{a}(x)\right\|-\frac{1}{l} \sum_{k=0}^{l-1} \frac{1}{n} \log \left\|M_{n}^{a}(x+k \omega)\right\|\right| \leq \frac{\delta}{2}
$$

for all $x$ except for a set of measure less than $\exp (-c l)$. Hence

$$
\begin{aligned}
\operatorname{mes} & \left\{x \in \mathbb{\mathbb { N }}:\left|\frac{1}{n} \log \left\|M_{n}^{a}(x)\right\|-\frac{1}{l} \sum_{k=0}^{l-1} \frac{1}{n} \log \left\|M_{n}^{a}(x+k \omega)\right\|\right|>\frac{\delta}{2}\right\} \\
& <\exp (-c l) \\
& <\exp \left(-c_{1} \delta n\right),
\end{aligned}
$$

where $c_{1}=c /\left(2 C_{1}\right)$.

From Theorem 2.1 we have

$$
\begin{aligned}
\operatorname{mes} & \left\{x \in \mathbb{T}:\left|\frac{1}{l} \sum_{k=0}^{l-1} \frac{1}{n} \log \left\|M_{n}^{a}(x+k \omega)\right\|-L_{n}^{a}\right|>\frac{\delta}{2}\right\} \\
& <\exp \left(-c \frac{\delta}{2} l+C(\log l)^{p}\right) \\
& <\exp \left(-c_{2} \delta^{2} n+C^{\prime}(\log n)^{p}\right) .
\end{aligned}
$$

Recall that Lemma 3.4 ensures that $c$ and $C$ don't depend on $n$.

Now (3.9) becomes

$$
\begin{aligned}
\operatorname{mes}\{x & \left.\in \mathbb{T}:\left|\log \left\|M_{n}^{a}(x)\right\|-n L_{n}^{a}\right|>n \delta\right\} \\
& <\exp \left(-c_{1} \delta n\right)+\exp \left(-c_{2} \delta^{2} n+C^{\prime}(\log n)^{p}\right) \\
& <2 \exp \left(-c \delta^{2} n+C^{\prime}(\log n)^{p}\right) \\
& <\exp \left(-c \delta^{2} n+C^{\prime \prime}(\log n)^{p}\right),
\end{aligned}
$$

where $c=c\left(c_{1}, c_{2}, \delta_{0}\right)$. This concludes the proof.

The same proof, with an adequate change in the second to last inequality, yields that for $\delta \geq \delta_{0}$ we have

$$
\operatorname{mes}\left\{x \in \mathbb{T}:\left|\log \left\|M_{n}^{a}(x)\right\|-n L_{n}^{a}\right|>n \delta\right\}<\exp \left(-c_{0} n \delta+C_{0}(\log n)^{p}\right) .
$$

Note that for $\delta_{0}$ large enough the above estimate, with $C_{0}=0$, also follows from (3.4). Also note that to get an estimate when $n=1$ one just needs to apply Theorem 2.1.

The same type of estimate holds for $M_{n}^{u}$ and $M_{n}$. 
Corollary 3.6. Let $\delta_{0}>0$. For any $\delta \in\left(0, \delta_{0}\right)$ and any integer $n>1$ we have

$$
\operatorname{mes}\left\{x \in \mathbb{T}:\left|\log \left\|M_{n}^{u}(x)\right\|-n L_{n}^{u}\right|>n \delta\right\}<\exp \left(-c_{0} n \delta^{2}+C_{0}(\log n)^{p}\right)
$$

where

$$
c_{0}=c_{0}\left(\|a\|_{\infty},\|b\|_{*},|E|, \omega, \delta_{0}\right)
$$

and

$$
C_{0}=C_{0}\left(\|a\|_{\infty},\|b\|_{*},|E|, \omega, p, \delta_{0}\right) .
$$

An analogous estimate holds for $M_{n}$.

Proof. Using (2.9) we easily get

$$
\begin{aligned}
\operatorname{mes}\{x & \left.\in \mathbb{T}:\left|\log \left\|M_{n}^{u}(x)\right\|-n L_{n}^{u}\right|>n \delta\right\} \\
\leq & \operatorname{mes}\left\{x \in \mathbb{T}:|\log |\left|M_{n}^{a}(x) \|-n L_{n}^{a}\right|>\frac{n \delta}{2}\right\} \\
& +\operatorname{mes}\left\{x \in \mathbb{T}:\left|\widetilde{S}_{n}(x)-n \widetilde{D}\right|>\frac{n \delta}{2}\right\} \\
& +\operatorname{mes}\left\{x \in \mathbb{T}:\left|S_{n}(x+\omega)-n D\right|>\frac{n \delta}{2}\right\} .
\end{aligned}
$$

The conclusion now follows from Lemma 3.5 and Theorem 2.1. The estimate for $M_{n}$ follows in the same way starting from (2.8).

The next step is to make use of the Avalanche Principle to improve the previous estimate. The following lemma is the most general application of the Avalanche Principle that suits our purposes.

Lemma 3.7. Let $n>1$ be an integer and $n=\sum_{j=1}^{m} l_{j}$ where $l_{j}$ are positive integers such that $l \leq l_{j} \leq 3 l$, with $l=l(n)$ a real number. Let $A_{j}(x)=A_{j}(x, n)$ be $2 \times 2$ matrices for $x \in \mathbb{T}$, and let $L_{k}, k \geq 1$ be a sequence of real numbers. If

$$
\begin{gathered}
l>\frac{2}{\gamma} \log n, \\
L_{l_{j}}-L_{l_{j}+l_{j+1}} \leq \frac{\gamma}{100}, L_{l_{j+1}}-L_{l_{j}+l_{j+1}} \leq \frac{\gamma}{100}, \quad j=1, \ldots, m-1, \\
\max _{1 \leq j \leq m}\left|\operatorname{det} A_{j}(x)\right| \leq 1, \quad \text { for a.e. } x \in \mathbb{T}, \\
\operatorname{mes}\left\{x \in \mathbb{T}:\left|\frac{1}{l_{j}} \log \left\|A_{j}(x)\right\|-L_{l_{j}}\right|>\frac{\gamma}{100}\right\} \leq \exp \left(-c_{0} l_{j}^{\sigma}\right), \quad j=1, \ldots, m,
\end{gathered}
$$


and

$$
\begin{aligned}
& \operatorname{mes}\left\{x \in:\left|\frac{1}{l_{j}+l_{j+1}} \log \left\|A_{j+1}(x) A_{j}(x)\right\|-L_{l_{j}+l_{j+1}}\right|>\frac{\gamma}{100}\right\} \\
& \quad \leq \exp \left(-c_{0}\left(l_{j}+l_{j+1}\right)^{\sigma}\right), \quad j=1, \ldots, m-1,
\end{aligned}
$$

then there exists an absolute constant $C_{0}$ such that

$$
\begin{aligned}
\mid \log & \left\|A_{m}(x) \ldots A_{1}(x)\right\|+\sum_{j=2}^{m-1} \log \left\|A_{j}(x)\right\|-\sum_{j=1}^{m-1} \log \left\|A_{j+1}(x) A_{j}(x)\right\| \mid \\
& <C_{0} m \exp \left(-\frac{\gamma}{2} l\right) \\
& <C_{0} \frac{1}{l}
\end{aligned}
$$

up to a set of measure less than $3 n \exp \left(-c_{0} l^{\sigma}\right)$.

Proof. Let $\mu=\exp (l \gamma / 2)$. We have

$$
\min _{1 \leq j \leq m}\left\|A_{j}(x)\right\| \geq \min _{1 \leq j \leq m} \exp \left(l_{j} L_{l_{j}}-\frac{\gamma}{100}\right)>\exp (l \gamma / 2)=\mu>n
$$

and

$$
\begin{aligned}
& \max _{1 \leq j<m-1}\left[\log \left\|A_{j+1}(x)\right\|+\log \left\|A_{j}(x)\right\|-\log \left\|A_{j+1}(x) A_{j}(x)\right\|\right] \\
& \quad \leq l_{j+1}\left(L_{l_{j+1}}+\frac{\gamma}{100}\right)+l_{j}\left(L_{l_{j}}+\frac{\gamma}{100}\right)-\left(l_{j}+l_{j+1}\right)\left(L_{l_{j+1}+l_{j}}-\frac{\gamma}{100}\right) \\
& \quad=l_{j+1}\left(L_{l_{j+1}}-L_{l_{j+1}+l_{j}}+\frac{2 \gamma}{100}\right)+l_{j}\left(L_{l_{j}}-L_{l_{j+1}+l_{j}}+\frac{2 \gamma}{100}\right) \\
& \quad<6 l \frac{3 \gamma}{100}<\frac{\gamma l}{4}=\frac{1}{2} \log \mu
\end{aligned}
$$

up to a set of measure $3 m \exp \left(-c_{0} l^{\sigma}\right)<3 n \exp \left(-c_{0} l^{\sigma}\right)$. The conclusion follows from the Avalanche Principle and the fact that $m / \mu<1 / l$.

As mentioned before, it is important for us that the constants in the deviations estimate can be chosen uniformly for $E$ in a compact set. For this we need to provide a bound for $L_{n}^{u}-L^{u}$ that holds for all $E$ in a compact set. First we state a simple estimate that we will use to deal with the integrals over the exceptional sets for our functions. 
Lemma 3.8. Let $f$ be a measurable function defined on $\mathbb{T}$ such that for any $\delta \geq \delta_{0}$ we have that $|f(x)| \leq \delta$ up to a set of measure less than $\exp \left(-c_{0} \delta\right)$. Then

$$
\|f\|_{L^{2}(\mathbb{T})} \leq C_{0},
$$

where

$$
C_{0}=C_{0}\left(c_{0}, \delta_{0}\right)
$$

Lemma 3.9. For any integer $n>1$ we have

$$
0 \leq L_{n}-L=L_{n}^{u}-L^{u}=L_{n}^{a}-L^{a}<C_{0} \frac{(\log n)^{2}}{n}
$$

where

$$
C_{0}=C_{0}\left(\|a\|_{\infty},\|b\|_{*},|E|, \omega, \gamma\right) .
$$

Proof. It is sufficient to get the estimate for large $n$. We will tacitly assume that $n$ is large enough for our estimates to hold. We should keep in mind that the choice of large $n$ should be uniform for $E$ in a bounded set.

It is easy to see that the conclusion follows if we have

$$
\left|L_{2 n}^{a}-L_{n}^{a}\right| \leq C \frac{(\log n)^{2}}{n} .
$$

Since we have

$$
\left|L_{2 n}^{a}-L_{n}^{a}\right|=\left|\int_{\mathbb{T}} \frac{\log \left\|M_{2 n}^{a}(x)\right\|-\log \left\|M_{n}^{a}(x+n \omega)\right\|-\log \left\|M_{n}^{a}(x)\right\|}{2 n} d x\right|,
$$

it will be sufficient to prove that

$$
\left|\log \left\|M_{2 n}^{a}(x)\right\|-\log \left\|M_{n}^{a}(x+n \omega)\right\|-\log \left\|M_{n}^{a}(x)\right\|\right| \leq C_{1}(\log n)^{2}
$$

up to a set not exceeding $C_{2} n^{-1}$ in measure. Indeed, from (3.1) it follows that for $\delta \geq \delta_{0}$ we have

$$
\left|\frac{\log \left\|M_{2 n}^{a}(x)\right\|-\log \left\|M_{n}^{a}(x+n \omega)\right\|-\log \left\|M_{n}^{a}(x)\right\|}{2 n}\right| \leq \delta
$$

up to a set not exceeding $\exp \left(-c_{1} \delta 2 n\right)+2 \exp \left(-c_{1} \delta n\right)<\exp (-c \delta n)$ in measure, and by using (3.11) and Lemma 3.8 we get

$$
\begin{aligned}
\left|L_{2 n}^{a}-L_{n}^{a}\right| & \leq \int_{\mathbb{T}}\left|\frac{\log \left\|M_{2 n}^{a}(x)\right\|-\log \left\|M_{n}^{a}(x+n \omega)\right\|-\log \left\|M_{n}^{a}(x)\right\|}{2 n}\right| d x \\
& \leq C_{1}(\log n)^{2}+C_{3} \sqrt{C_{2} n^{-1}} \\
& \leq C(\log n)^{2} .
\end{aligned}
$$


Now we check that the sufficient condition (3.11) holds. Let $l=\left[C_{l} \log n\right]$ and $m=[n / l]$. If $C_{l}$ is sufficiently large, we have $l>2 \log n / \gamma$ and $3 n \exp (-c l)<n^{-1}$. We want to choose $C_{l}$ so that $L_{l}^{u}-L_{2 l}^{u} \leq \gamma / 100$ and $C_{l} \leq C$ (note that without the bound, such $C_{l}$ obviously exists). Suppose that $L_{2^{j} l}^{u}-L_{2^{j+1} l}^{u}>\frac{\gamma}{100}$ for $j \geq 0$. Then using (3.8) we get

$$
C-\frac{\tilde{D}+D}{2} \geq L_{l}^{u}-L_{2^{j+1} l}^{u}>\frac{j \gamma}{100}
$$

This shows that by eventually replacing $l$ with $2^{j} l$ with some

$$
j<100(2 C-\tilde{D}-D) / \gamma
$$

we will have $L_{l}^{u}-L_{2 l}^{u} \leq \gamma / 100$, and the corresponding $C_{l}$ will be bounded. Using Corollary 3.6 and Lemma 3.7 we get

$$
\left|\log \left\|M_{m l}^{a}(x)\right\|+\sum_{j=1}^{m-2} \log \left\|M_{l}^{a}(x+j l \omega)\right\|-\sum_{j=0}^{m-2} \log \left\|M_{2 l}^{a}(x+j l \omega)\right\|\right|<C
$$

up to a set not exceeding $n^{-1}$ in measure, and analogous estimates for $\log \| M^{a}(x+$ $m l \omega) \|$ and $\log \left\|M_{2 m l}^{a}(x)\right\|$. Recall that we apply the Avalanche Principle to $M_{n}^{u}$ but the conclusion also holds for $M_{n}^{a}$. Note that we need to have $m \geq 2$. This clearly holds for large enough $n$ depending on $C_{l}$. This can be done uniformly for $E$ in a bounded set because of our bound on $C_{l}$. Putting these estimates together we get

$$
\begin{aligned}
\mid \log & \left\|M_{2 m l}^{a}(x)\right\|-\log \left\|M_{m l}^{a}(x+m l \omega)\right\|-\log \left\|M_{m l}^{a}(x)\right\| \\
& +\log \left\|M_{l}^{a}(x+(m-1) l \omega)\right\|+\log \left\|M_{l}^{a}(x+m l \omega)\right\| \\
& \quad-\log \left\|M_{2 l}^{a}(x+(m-1) l \omega)\right\| \mid \\
< & C
\end{aligned}
$$

up to a set not exceeding $C n^{-1}$ in measure. By (3.1) we have that

$$
\left|\log \left\|M_{l}^{a}(x)\right\|\right| \leq C \log n
$$

up to a set not exceeding $n^{-1}$ in measure. From this, similar estimates, and (3.13) we get

$$
\left|\log \left\|M_{2 m l}^{a}(x)\right\|-\log \left\|M_{m l}^{a}(x+m l \omega)\right\|-\log \left\|M_{m l}^{a}(x)\right\|\right|<C \log n
$$

up to a set not exceeding $C n^{-1}$ in measure.

From (3.5) we get that for sufficiently large $\delta$ we have

$$
\left|\log \left\|M_{n}^{a}(x)\right\|-\log \left\|M_{m l}^{a}(x)\right\|\right| \leq \delta(n-m l)
$$

up to a set not exceeding $\exp (-c \delta(n-m l))$ in measure. We can choose $\delta>(\log n) / c$ to conclude that

$$
\left|\log \left\|M_{n}^{a}(x)\right\|-\log \left\|M_{m l}^{a}(x)\right\|\right| \leq C(\log n)^{2}
$$


up to a set not exceeding $n^{-1}$ in measure. From this, similar estimates (using (3.5) and (3.6)), and (3.14) we can conclude that

$$
\left|\log \left\|M_{2 n}^{a}(x)\right\|-\log \left\|M_{n}^{a}(x+n \omega)\right\|-\log \left\|M_{n}^{a}(x)\right\|\right|<C(\log n)^{2}
$$

up to a set not exceeding $C n^{-1}$ in measure. Thus we proved (3.11) and this concludes the proof.

The bound from the previous lemma can be improved, as in [4], Theorem 5.1, to $L_{n}-L \leq C_{0} / n$. However, we won't need this better bound in this paper.

Now we are able to prove the improved version of the deviations estimate (cf. [4], Theorem 7.1). A couple of other deviations estimates exist for the Jacobi case. Kai Tao proved an estimate which is very similar to Lemma 3.5 (see [10], Theorem 2.15). Jitomirskaya, Koslover, and Schulteis generalized the estimate [2], Lemma 4, to the Jacobi case (see [7], Lemma 1; also see [8], Theorem 3.1, for a more general version). Their estimate is given in terms of approximants of the frequency, and in particular it applies to any irrational frequency. As in the Schrödinger case, the fact that we are restricting to frequencies satisfying a strong Diophantine condition allows us to obtain a better estimate.

Theorem 3.10. For any $\delta>0$ and any integer $n>1$ we have

$$
\operatorname{mes}\left\{x \in \mathbb{T}:|\log |\left|M_{n}^{a}(x) \|-n L_{n}^{a}\right|>\delta n\right\}<\exp \left(-c_{0} \delta n+C_{0}(\log n)^{p}\right)
$$

where

$$
c_{0}=c_{0}\left(\|a\|_{\infty},\|b\|_{*},|E|, \omega, \gamma\right)
$$

and

$$
C_{0}=C_{0}\left(\|a\|_{\infty},\|b\|_{*},|E|, \omega, \gamma, p\right) .
$$

The same estimate, with possibly different constants, holds with $L^{a}$ instead of $L_{n}^{a}$.

Proof. First note that due to (3.4) we just need to check the estimate for $\delta<\delta_{0}$. Furthermore, note that the estimate is trivial if $-c_{0} \delta n+C_{0}(\log n)^{p}>0$. Hence we just need to check the estimate for $\delta$ satisfying

$$
C \frac{(\log n)^{p}}{n} \leq \delta<\delta_{0},
$$

where $C=C_{0} / c_{0}$ can be made as large as we need by choosing $C_{0}$ large. Furthermore by choosing $C_{0}$ large enough we can make sure that the deviations estimate holds trivially for small $n$. Hence it is enough to check the estimate for $n$ large enough. 
Let $l=[\delta n]+1, m=[n / l]$ and $l^{\prime}=n-(m-1) l$. An application of the Avalanche Principle (using Corollary 3.6, (3.15), and Lemma 3.7) yields

$$
\begin{aligned}
\log \| & M_{n}^{a}(x)\left\|+\sum_{j=1}^{m-2} \log \right\| M_{l}^{a}(x+j l \omega)\|-\log \| M_{l^{\prime}+l}^{a}(x+(m-2) l \omega) \| \\
& -\sum_{j=0}^{m-3} \log \left\|M_{l}^{a}(x+(j+1) l \omega) M_{l}^{a}(x+j l \omega)\right\| \\
= & O\left(\frac{1}{l}\right)
\end{aligned}
$$

up to a set of measure less than $3 n \exp (-c l)<\exp (-c \delta n / 2)$. From (3.1) we can conclude that

$$
\begin{aligned}
\mid \log & \left\|M_{l^{\prime}}^{a}(x+(m-1) l \omega) M_{l}^{a}(x+(m-2) l \omega)\right\| \mid \\
\quad & =\left|\log \left\|M_{l^{\prime}+l}^{a}(x+(m-2) l \omega)\right\|\right| \\
& \leq C l
\end{aligned}
$$

up to a set of measure less than $\exp (-c l) \leq \exp (-c \delta n)$. Hence

$$
\log \left\|M_{n}^{a}(x)\right\|+\sum_{j=1}^{m-2} \log \left\|M_{l}^{a}(x+j l \omega)\right\|-\sum_{j=0}^{m-3} \log \left\|M_{2 l}^{a}(x+j l \omega)\right\|=O(l)
$$

up to a set of measure less than $\exp (-c \delta n)$. Summing the above estimate with $x+k \omega$ instead of $x$ yields

$$
\begin{aligned}
& \frac{1}{l} \sum_{k=0}^{l-1} \log \left\|M_{n}^{a}(x+k \omega)\right\|+\sum_{j=l}^{(m-1) l-1} \frac{1}{l} \log \left\|M_{l}^{a}(x+j \omega)\right\| \\
& \quad-\sum_{j=0}^{(m-2) l-1} \frac{1}{l} \log \left\|M_{2 l}^{a}(x+j \omega)\right\| \\
& =O(l)
\end{aligned}
$$

up to a set of measure less than $l \exp (-c \delta n)<\exp (-c \delta n / 2)$. Using (3.7) we can conclude that

$$
\begin{aligned}
& \log \left\|M_{n}^{a}(x)\right\|+\sum_{j=l}^{(m-1) l-1} \frac{1}{l} \log \left\|M_{l}^{a}(x+j \omega)\right\|-\sum_{j=0}^{(m-2) l-1} \frac{1}{l} \log \left\|M_{2 l}^{a}(x+j \omega)\right\| \\
& =O(l)
\end{aligned}
$$


up to a set of measure less than $\exp \left(-c_{1} \delta n\right)+\exp \left(-c_{2} l\right)<\exp (-c \delta n)$. From this, Theorem 2.1, and (3.8) it follows that

$$
\log \left\|M_{n}^{a}(x)\right\|+(m-2) l\left(L_{l}^{a}-2 L_{2 l}^{a}\right)=O(\delta n)
$$

up to a set of measure less than

$$
2 \exp \left(-c_{1} \delta n+C(\log n)^{p}\right)+\exp \left(-c_{2} \delta n\right)<\exp \left(-c \delta n+C(\log n)^{p}\right) .
$$

Integrating over $\mathbb{T}$ and using Lemma 3.8 yields

$$
\begin{aligned}
\left|n L_{n}^{a}+(m-2) l\left(L_{l}^{a}-2 L_{2 l}^{a}\right)\right| & <C_{1} \delta n+C_{2} n \exp \left(\left(-c \delta n+C(\log n)^{p}\right) / 2\right) \\
& <C \delta n .
\end{aligned}
$$

Note that for the last inequality to hold we need to choose $C$ large enough in (3.15). Now we have that

$$
\left|\log \left\|M_{n}^{a}(x)\right\|-n L_{n}^{a}\right|<C \delta n
$$

up to a set of measure less than $\exp \left(-c \delta n+C(\log n)^{p}\right)$. The fact that $L_{n}^{a}$ can be replaced by $L^{a}$ follows from Lemma 3.9 and (3.15).

Corollary 3.11. For any $\delta>0$ and any integer $n>1$ we have

$$
\operatorname{mes}\left\{x \in \mathbb{T}:|\log |\left|M_{n}^{u}(x) \|-n L_{n}^{u}\right|>\delta n\right\}<\exp \left(-c_{0} \delta n+C_{0}(\log n)^{p}\right)
$$

where

and

$$
c_{0}=c_{0}\left(\|a\|_{\infty},\|b\|_{*},|E|, \omega, \gamma\right)
$$

$$
C_{0}=C_{0}\left(\|a\|_{\infty},\|b\|_{*},|E|, \omega, \gamma, p\right) .
$$

The same estimate, with possibly different constants, holds with $L^{u}$ instead of $L_{n}^{u}$. An analogous statement holds for $M_{n}$.

Proof. The proof is the same as for Corollary 3.6.

Next we establish some estimates that will be needed in the next section. First we prove a uniform upper bound for $\log \left\|M_{n}^{a}\right\|$. We will need the following general result about averages of subharmonic functions.

Lemma 3.12 ([5], Lemma 4.1). Let $u$ be a subharmonic function and let

$$
u(z)=\int_{\mathbb{C}} \log |z-\zeta| d \mu(\zeta)+h(z)
$$

be its Riesz representation on a neighborhood of $\mathcal{A}_{\rho}$. If $\mu\left(\mathcal{A}_{\rho}\right)+\|h\|_{L^{\infty}\left(\mathcal{A}_{\rho}\right)} \leq M$, then for any $r_{1}, r_{2} \in(1-\rho, 1+\rho)$ we have

$$
\left|\left\langle u\left(r_{1}(\cdot)\right)\right\rangle-\left\langle u\left(r_{2}(\cdot)\right)\right\rangle\right| \leq C_{0}\left|r_{1}-r_{2}\right|,
$$

where $C_{0}=C_{0}(M, \rho)$. 
The following corollary is an immediate consequence of the previous lemma and Lemma 3.4.

Corollary 3.13. There exists a constant

$$
C_{0}=C_{0}\left(\|a\|_{\infty},\|b\|_{*},|E|, \rho_{0}, \rho_{0}^{\prime}, \rho_{0}^{\prime \prime}\right)
$$

such that

$$
\left|L_{n}^{u}\left(r_{1}\right)-L_{n}^{u}\left(r_{2}\right)\right|=\left|L_{n}^{a}\left(r_{1}\right)-L_{n}^{a}\left(r_{2}\right)\right| \leq C_{0}\left|r_{1}-r_{2}\right|
$$

for any $r_{1}, r_{2} \in\left(1-\rho_{0}, 1+\rho_{0}\right)$ and any positive integer $n$.

Proposition 3.14. For any integer $n>1$ we have that

$$
\sup _{x \in \mathbb{T}} \log \left\|M_{n}^{a}(x)\right\| \leq n L_{n}^{a}+C_{0}(\log n)^{p}
$$

where

$$
C_{0}=C_{0}\left(\|a\|_{\infty},\|b\|_{*},|E|, \omega, \gamma, p\right) .
$$

Proof. It is sufficient to establish the estimate for large $n$. From the large deviations estimate, with $n \delta=C(\log n)^{p}$ where $C$ is sufficiently large, we have

$$
\log \left\|M_{n}^{a}(r x)\right\|-n L_{n}^{a}(r) \leq C(\log n)^{p}
$$

except for a set $\mathscr{B}(r)$ of measure less than

$$
\exp \left(-c_{1} C(\log n)^{p}+C^{\prime}(\log n)^{p}\right)<\exp \left(-c(\log n)^{p}\right) .
$$

Here $r$ is in a neighborhood of 1 such that $L^{u}(r) \geq \gamma / 2$. Such a neighborhood exists because of Corollary 3.13. By the subharmonicity of $\log \left\|M_{n}^{a}(z)\right\|$ we have

$$
\begin{aligned}
\log \left\|M_{n}^{a}(x)\right\|-n L_{n}^{a} & \leq \frac{1}{\pi n^{-2}} \int_{D\left(x, n^{-1}\right)}\left(\log \left\|M_{n}^{a}(z)\right\|-n L_{n}^{a}\right) d A(z) \\
& \leq \frac{1}{\pi n^{-2}} \int_{1-n^{-1}}^{1+n^{-1}} \int_{x-2 n^{-1}}^{x+2 n^{-1}}\left|\log \left\|M_{n}^{a}(r y)\right\|-L_{n}^{a}\right| r d y d r .
\end{aligned}
$$

For $r \in\left(1-n^{-1}, 1+n^{-1}\right)$ we have

$$
\begin{aligned}
& \int_{x-2 n^{-1}}^{x+2 n^{-1}}\left|\log \left\|M_{n}^{a}(r y)\right\|-L_{n}^{a}\right| d y \\
& \quad \leq \int_{x-2 n^{-1}}^{x+2 n^{-1}}\left|\log \left\|M_{n}^{a}(r y)\right\|-L_{n}^{a}(r)\right| d y+\left|L_{n}^{a}-L_{n}^{a}(r)\right| \\
& \quad \leq C_{1}(\log n)^{p} n^{-1}+C_{2} n \exp \left(-c \frac{(\log n)^{p}}{2}\right)+C_{3} n^{-1} \\
& \quad<C(\log n)^{p} n^{-1} .
\end{aligned}
$$


As usual, we used Lemma 3.8 to deal with the exceptional set. Plugging this estimate in (3.16) yields the desired conclusion.

As was mentioned in the introduction, from this point forward we will make use of the fact that $\tilde{b}=\bar{b}$ on $\mathbb{T}$. In particular we will tacitly use that $D=\widetilde{D}, S=\widetilde{S}$, $L_{n}=L_{n}^{u}, L=L^{u}$, and $|\tilde{b}|=|\bar{b}|=|b|$.

Next we want to estimate $L_{n}(E)-L_{n}\left(E_{0}\right)$ in a neighborhood of $E_{0}$.

Lemma 3.15. There exist constants

$$
C_{0}=C_{0}\left(\|a\|_{\infty},\|b\|_{*}, \max \left\{\left|E_{1}\right|,\left|E_{2}\right|\right\}\right)
$$

and

$$
c_{0}=c_{0}\left(\|b\|_{*}, \omega\right)
$$

such that

$$
\begin{aligned}
& \left|\log \left\|M_{l}^{u}\left(x, E_{1}\right)\right\|-\log \left\|M_{l}^{u}\left(x, E_{2}\right)\right\|\right| \\
& \quad=\left|\log \left\|M_{l}^{a}\left(x, E_{1}\right)\right\|-\log \left\|M_{l}^{a}\left(x, E_{2}\right)\right\|\right| \\
& \quad \leq \exp \left(C_{0} l\right)\left|E_{1}-E_{2}\right|
\end{aligned}
$$

holds for any positive integer $l$ and any $x$ up to a set (independent of $E_{1}$ and $E_{2}$ ) of measure less than $\exp \left(-c_{0} l\right)$.

Proof. The identity follows from (2.9). By the Mean Value Theorem we have

$$
\begin{aligned}
& \left|\log \left\|M_{l}^{a}\left(x, E_{1}\right)\right\|-\log \left\|M_{l}^{a}\left(x, E_{2}\right)\right\|\right| \\
& \quad \leq \frac{1}{\min \left\{\left\|M_{l}^{a}\left(x, E_{1}\right)\right\|,\left\|M_{l}^{a}\left(x, E_{2}\right)\right\|\right\}}\left|\left\|M_{l}^{a}\left(x, E_{1}\right)\right\|-\left\|M_{l}^{a}\left(x, E_{2}\right)\right\|\right| \\
& \quad \leq \frac{1}{\min \left\{\left\|M_{l}^{a}\left(x, E_{1}\right)\right\|,\left\|M_{l}^{a}\left(x, E_{2}\right)\right\|\right\}} \sup _{E \in\left[E_{1}, E_{2}\right]}\left\|\frac{\partial}{\partial E} M_{l}^{a}(x, E)\right\|\left|E_{1}-E_{2}\right| .
\end{aligned}
$$

There exists a constant

$$
C=C\left(\|a\|_{\infty},\|b\|_{\infty}, \max \left\{\left|E_{1}\right|,\left|E_{2}\right|\right\}\right)
$$

such that

$$
\sup _{E \in\left[E_{1}, E_{2}\right]}\left\|\frac{\partial}{\partial E} M_{l}^{a}(x, E)\right\| \leq \exp (C l) .
$$

The conclusion now follows by using (3.1). 
Lemma 3.16. Fix $E_{0} \in \mathbb{C}$ such that $L\left(E_{0}\right) \geq \gamma$. There exist constants

and

$$
\begin{aligned}
& C_{0}=C_{0}\left(\|a\|_{\infty},\|b\|_{*},\left|E_{0}\right|, \omega, \gamma\right), \\
& C_{1}=C_{1}\left(\|a\|_{\infty},\|b\|_{*},\left|E_{0}\right|, \omega, \gamma\right),
\end{aligned}
$$

$$
n_{0}=n_{0}\left(\|a\|_{\infty},\|b\|_{*},\left|E_{0}\right|, \omega, \gamma\right)
$$

such that we have

$$
\left|\log \left\|M_{n}^{a}(x, E)\right\|-\log \left\|M_{n}^{a}\left(x, E_{0}\right)\right\|\right| \leq n^{-C_{0}}
$$

for $n \geq n_{0},\left|E-E_{0}\right|<n^{-C_{1}}$, and all $x$ up to a set $\mathcal{B}=\mathcal{B}\left(n, E_{0}\right)$ of measure less than $n^{-1}$.

Proof. Let $l=\left[C_{2} \log n\right], m=[n / l]$, and $l^{\prime}=n-(m-2) l$. In what follows we should keep in mind that some of the estimates hold by choosing $C_{2}$ large enough. To be able to apply the Avalanche Principle we will need that $m \geq 2$, hence we also need that $n$ is large enough. Applying the Avalanche Principle (see Lemma 3.7) we get

$$
\begin{aligned}
\log \| & M_{n}^{a}\left(x, E_{0}\right)\left\|+\sum_{j=1}^{m-2} \log \right\| M_{l}^{a}\left(x+j l \omega, E_{0}\right) \| \\
& \quad-\log \left\|M_{l+l^{\prime}}^{a}\left(x+(m-2) l \omega, E_{0}\right)\right\|-\sum_{j=0}^{m-3} \log \left\|M_{2 l}^{a}\left(x+j l \omega, E_{0}\right)\right\| \\
= & O\left(\frac{n}{l} \exp \left(-\frac{\gamma}{2} l\right)\right) \\
= & O\left(\frac{1}{n^{c C_{2}}}\right)
\end{aligned}
$$

up to a set of measure $3 n \exp \left(-c_{1} l\right)<n^{-c C_{2}}$. We claim that the Avalanche Principle can be applied, with the same $\mu$, for the same factorization of $M_{n}^{a}(x, E)$. Note that we cannot apply the deviations estimate since we don't know whether $L(E)>0$. For example, Lemma 3.15 and Corollary 3.6 imply that

$$
\begin{aligned}
\log \left\|M_{l}^{u}(x, E)\right\| & \geq \log \left\|M_{l}^{u}\left(x, E_{0}\right)\right\|-\exp \left(C l-C_{1} \log n\right) \\
& \geq\left(\gamma-\frac{\gamma}{100}\right) l-\exp \left(C l-C_{1} \log n\right) \\
& >\frac{\gamma}{2} l
\end{aligned}
$$

up to a set of measure $\exp \left(-c_{1} l\right)+\exp \left(-c_{2} l\right)<\exp (-c l)$. Note that the exceptional set from the deviation estimate is already included in the exceptional set for (3.17) 
and recall that the exceptional set from Lemma 3.15 doesn't depend on $E$. Also note that $C_{1}$ needs to satisfy $C_{1} \geq C C_{2}$. The other estimates needed for the Avalanche Principle are obtained similarly, provided $C_{1}$ is large enough. Hence, (3.17) holds with $E$ instead of $E_{0}$. The conclusion follows by subtracting (3.17) for $E$ and $E_{0}$ and using Lemma 3.15 (again, $C_{1}$ needs to be chosen to be large enough).

Corollary 3.17. Fix $E_{0} \in \mathbb{C}$ such that $L\left(E_{0}\right) \geq \gamma$. There exist constants

and

$$
\begin{aligned}
& C_{0}=C_{0}\left(\|a\|_{\infty},\|b\|_{*},\left|E_{0}\right|, \omega, \gamma\right), \\
& C_{1}=C_{1}\left(\|a\|_{\infty},\|b\|_{*},\left|E_{0}\right|, \omega, \gamma\right),
\end{aligned}
$$

$$
n_{0}=n_{0}\left(\|a\|_{\infty},\|b\|_{*},\left|E_{0}\right|, \omega, \gamma\right)
$$

such that we have

$$
\left|n\left(L_{n}(E)-L_{n}\left(E_{0}\right)\right)\right|=\left|n\left(L_{n}^{a}(E)-L_{n}^{a}\left(E_{0}\right)\right)\right| \leq n^{-C_{0}}
$$

for $n \geq n_{0}$ and $\left|E-E_{0}\right|<n^{-C_{1}}$.

Proof. Integrate the estimate of the previous lemma. To deal with the exceptional set we used Lemma 3.8 and the fact that as a consequence of (3.1) we have

$$
\left|\log \left\|M_{n}^{a}(x, E)\right\|-\log \left\|M_{n}^{a}\left(x, E_{0}\right)\right\|\right| \leq \lambda n
$$

up to a set of size $\exp (-c \lambda n)$ for any $\lambda \geq \lambda_{0}$.

\section{Estimates for the entries of the fundamental matrix}

We will need the following particular case of a lemma from [5].

Lemma 4.1 ([5], Lemma 2.4). Let u be a subharmonic function defined on $\mathcal{A}_{\rho}$ such that $\sup _{\mathcal{A}_{\rho}} u \leq M$. There exist constants $C_{1}=C_{1}(\rho)$ and $C_{2}$ such that, if for some $0<\delta<1$ and some L we have

$$
\operatorname{mes}\{x \in \mathbb{T}: u(x)<-L\}>\delta,
$$

then

$$
\sup _{\mathbb{T}} u \leq C_{1} M-\frac{L}{C_{1} \log \left(C_{2} / \delta\right)} .
$$

Let $I_{a, E}=\int_{\mathbb{T}} \log |a(x)-E| d x$. Note that $\left|I_{a, E}\right|<\infty$ if and only if $a \not \equiv E$. If $a \equiv E$ then it is straightforward to see that $L=0$. Hence if $L(E)>0$ then $\left|I_{a, E}\right|<\infty$. Furthermore, if $L(E)>0$ on some set, it can be seen that $I_{a, E}$ is continuous in $E$ on that set. 
Lemma 4.2. There exists $l_{0}=l_{0}\left(\|a\|_{\infty}, I_{a, E},\|b\|_{*},|E|, \omega, \gamma\right)$ such that

$$
\operatorname{mes}\left\{x \in \mathbb{T}:\left|f_{l}(x)\right| \leq \exp \left(-l^{3}\right)\right\} \leq \exp (-l)
$$

for all $l \geq l_{0}$.

Proof. We argue by contradiction. Assume

$$
\operatorname{mes}\left\{x \in \mathbb{T}:\left|f_{l}(x)\right| \leq \exp \left(-l^{3}\right)\right\}>\exp (-l)
$$

for arbitrarily large $l$. We will be tacitly using the fact that $l$ can be arbitrarily large. We have that

$$
\left|f_{l}^{a}(x)\right|=\left|f_{l}(x)\right| \prod_{j=1}^{l}|b(x+j \omega)| \leq \exp \left(-l^{3}\right) C^{l} \leq \exp \left(-l^{3} / 2\right)
$$

on a set of measure greater than $\exp (-l)$. Hence

$$
\operatorname{mes}\left\{x \in \mathbb{T}:\left|f_{l}^{a}(x)\right| \leq \exp \left(-l^{3} / 2\right)\right\}>\exp (-l) .
$$

At the same time we have that

$$
\log \left|f_{l}^{a}(x)\right| \leq \log \left\|M_{l}^{a}(x)\right\| \leq C l
$$

for all $x$, so by applying Lemma 4.1 we get that

$$
\left|f_{l}^{a}(x)\right| \leq \exp \left(C_{1} l-\frac{l^{3}}{C_{2} \log \left(C_{3} \exp (l)\right)}\right) \leq \exp \left(-C l^{2}\right)
$$

for all $x$ and consequently

$$
\left|f_{l}(x)\right| \leq \exp \left(l(1-D)-C_{1} l^{2}\right) \leq \exp \left(-C l^{2}\right)
$$

for all $x$ except for a set of measure less than $\exp \left(-c_{1} l+r_{l}\right)<\exp (-c l)$.

From Corollary 3.11 we have that

$$
\begin{aligned}
\exp (l \gamma) \leq & \left\|M_{l}(x)\right\|^{2} \\
& \leq 2\left(\left|f_{l}(x)\right|^{2}+\left|f_{l-1}(x)\right|^{2}+\left|\frac{b(x)}{b(x+\omega)} f_{l-1}(x+\omega)\right|^{2}\right. \\
& \left.\quad+\left|\frac{b(x)}{b(x+\omega)} f_{l-2}(x+\omega)\right|^{2}\right)
\end{aligned}
$$

for all $x$ except for a set of measure less than $\exp \left(-c_{1} \gamma l / 2+r_{l}\right)<\exp (-c l)$. Suppose that

$$
\left|\frac{b(x)}{b(x+\omega)} f_{l-1}(x+\omega)\right|^{2} \geq \frac{1}{4} \exp (l \gamma)
$$


for all $x$ except for a set of measure less than $1 / 3$ (any constant in $(0,1 / 2)$ would work). Since

$$
\begin{aligned}
\frac{\overline{b(x)}}{b(x+l \omega)} & =\operatorname{det} M_{l}(x) \\
& =-\frac{\overline{b(x)}}{b(x+\omega)} f_{l}(x) f_{l-2}(x+\omega)+\frac{\overline{b(x)}}{b(x+\omega)} f_{l-1}(x) f_{l-1}(x+\omega)
\end{aligned}
$$

it follows that

$$
\begin{aligned}
& \left|f_{l-1}(x)\right| \\
& \quad=\left|\frac{b(x)}{b(x+\omega)} f_{l-1}(x+\omega)\right|^{-1}\left|\frac{b(x)}{b(x+l \omega)}+\frac{b(x)}{b(x+\omega)} f_{l}(x) f_{l-2}(x+\omega)\right| \\
& \quad \leq 2 \exp \left(-\frac{l \gamma}{2}\right)\left(C_{1} \exp (\delta-D)+\exp \left(-C_{2} l^{2}+C_{3} l\right)\right)
\end{aligned}
$$

for all $x$ except for a set of measure less than $1 / 3+\exp \left(-c_{1} \delta+r_{1}\right)+\exp \left(-c_{1} l\right)+$ $\exp \left(-c_{2} l+r_{l}^{\prime}\right)$. For the first factor in the above estimate we used (4.3). For the first term in the second factor we applied Theorem 2.1 with $u(x)=b(x+l \omega)$ and $n=1$ to get

$$
\left|\frac{b(x)}{b(x+l \omega)}\right| \leq \frac{C_{1}}{|b(x+l \omega)|} \leq C_{1} \exp (\delta-D)
$$

up to a set of measure less than $\exp \left(-c_{1} \delta+r_{1}\right)$. For the second term in the second factor we used (4.1), and (2.1) (recall that $|b|=|\bar{b}|=|\tilde{b}|$ on $\mathbb{T}$ ) together with the deviations estimate for $M_{l}$ (with $\delta=1$ ) to conclude that

$$
\left|\frac{b(x)}{b(x+\omega)} f_{l-2}(x+\omega)\right| \leq\left\|M_{l}(x)\right\| \leq \exp ((L+1) l)=\exp \left(C_{3} l\right)
$$

up to a set of measure less than $\exp \left(-c_{2} l+r_{l}^{\prime}\right)$. Choosing $\delta=l \gamma / 2$ we get

$$
\left|f_{l-1}(x)\right| \leq C
$$

for all $x$ except for a set of measure less than $1 / 3+\exp (-c l)$. This contradicts (4.3) because

$$
\left|f_{l-1}(x+\omega)\right| \leq C
$$

and (4.3) would hold at the same time on a set of measure greater than 1/3-exp $(-c l)$. Hence we must have

$$
\left|\frac{b(x)}{b(x+\omega)} f_{l-1}(x+\omega)\right|^{2}<\frac{1}{4} \exp (l \gamma)
$$

on a set of measure greater than $1 / 3$. At the same time 


$$
\begin{aligned}
& \exp ((l+1) \gamma) \leq\left\|M_{l+1}(x)\right\|^{2} \\
& \leq 2\left(\left|f_{l+1}(x)\right|^{2}+\left|f_{l}(x)\right|^{2}+\left|\frac{b(x)}{b(x+\omega)} f_{l}(x+\omega)\right|^{2}\right. \\
& \left.+\left|\frac{b(x)}{b(x+\omega)} f_{l-1}(x+\omega)\right|^{2}\right)
\end{aligned}
$$

for all $x$ except for a set of measure less than

$$
\exp \left(-c_{1} \gamma(l+1) / 2+r_{l+1}\right)<\exp (-c l) .
$$

This, (4.1), and (4.4) imply that we must have

$$
\begin{aligned}
& \left|f_{l+1}(x)\right|^{2} \\
& \quad \geq \frac{1}{2} \exp ((l+1) \gamma)-\exp \left(-C_{1} l^{2}\right)-C_{2} \exp \left(l-D-C_{1} l^{2}\right)-\frac{1}{4} \exp (l \gamma) \\
& \quad>\frac{1}{4} \exp (l \gamma)
\end{aligned}
$$

on a set of measure greater than

$$
\frac{1}{3}-\exp \left(-c_{1} l\right)-2 \exp \left(-c_{2} l\right)-\exp \left(-c_{3} l+r_{1}\right)>\frac{1}{3}-\exp (-c l) .
$$

From

$$
\begin{aligned}
& \frac{\overline{b(x)}}{b(x+(l+1) \omega)} \\
& \quad=\operatorname{det} M_{l+1}(x) \\
& \quad=-\frac{\overline{b(x)}}{b(x+\omega)} f_{l+1}(x) f_{l-1}(x+\omega)+\frac{\overline{b(x)}}{b(x+\omega)} f_{l}(x) f_{l}(x+\omega)
\end{aligned}
$$

it can be seen that

$$
\begin{aligned}
& \left|\frac{b(x)}{b(x+\omega)} f_{l-1}(x+\omega)\right| \\
& \quad=\left|f_{l+1}(x)\right|^{-1}\left|\frac{b(x)}{b(x+(l+1) \omega)}-\frac{b(x)}{b(x+\omega)} f_{l}(x) f_{l}(x+\omega)\right| \\
& \quad \leq 2 \exp \left(-\frac{l \gamma}{2}\right)\left(C_{1} \exp (\delta-D)+C_{1} \exp \left(\delta-D-C_{2} l^{2}\right)\right)
\end{aligned}
$$

on a set of measure greater than $1 / 3-\exp \left(-c_{1} l\right)-2 \exp \left(-c_{2} \delta+r_{1}\right)-2 \exp \left(-c_{3} l\right)$.

Choosing $\delta=l \gamma / 5$ we get

$$
\left|\frac{b(x)}{b(x+\omega)} f_{l-1}(x+\omega)\right| \leq \exp \left(-\frac{l \gamma}{4}\right)
$$


on a set of measure greater than $1 / 3-\exp (-c l)$. We will contradict (4.2) by showing that

$$
\begin{aligned}
& \left|f_{l}(x)\right|^{2}+\left|f_{l-1}(x)\right|^{2}+\left|\frac{b(x)}{b(x+\omega)} f_{l-1}(x+\omega)\right|^{2}+\left|\frac{b(x)}{b(x+\omega)} f_{l-2}(x+\omega)\right|^{2} \\
& \quad \leq C
\end{aligned}
$$

on a set of measure greater than $1 / 3-\exp (-c l)$. Let $G_{l}$ be the set on which (4.5) holds.

By writing

$$
\begin{aligned}
& M_{l}(x+\omega) \\
& \quad=\frac{1}{b(x+(l+1) \omega)}\left[\begin{array}{cc}
a(x+l \omega)-E & -\overline{b(x+l \omega)} \\
b(x+(l+1) \omega) & 0
\end{array}\right] M_{l-1}(x+\omega)
\end{aligned}
$$

we get

$$
f_{l}(x+\omega)=\frac{a(x+l \omega)-E}{b(x+(l+1) \omega)} f_{l-1}(x+\omega)-\frac{\overline{b(x+l \omega)}}{b(x+(l+1) \omega)} f_{l-2}(x+\omega) .
$$

From this we deduce that

$$
\begin{aligned}
& \left|\frac{b(x)}{b(x+\omega)} f_{l-2}(x+\omega)\right| \\
& =\left|\frac{b(x+(l+1) \omega)}{b(x+l \omega)}\right| \\
& \quad \cdot\left|\frac{a(x+l \omega)-E}{b(x+(l+1) \omega)} b(x) b(x+\omega) f_{l-1}(x+\omega)-\frac{b(x)}{b(x+\omega)} f_{l}(x+\omega)\right| \\
& \leq C_{1} \exp (\delta-D)\left(C_{2} \exp (\delta-D-\gamma l / 4)+C_{1} \exp \left(\delta-D-C_{3} l^{2}\right)\right)
\end{aligned}
$$

on a subset of $G_{l}$ of measure greater than

$$
\frac{1}{3}-3 \exp \left(-c_{1} \delta+r_{1}\right)-\exp \left(-c_{2} l\right)-\exp \left(-c_{3} l\right) .
$$

By choosing $\delta=\gamma l / 17$ we get

$$
\left|\frac{b(x)}{b(x+\omega)} f_{l-2}(x+\omega)\right| \leq \exp \left(-\frac{\gamma l}{8}\right)
$$

on a subset of $G_{l}$ of measure greater than $1 / 3-\exp (-c l)$.

By writing

$$
M_{l}(x-\omega)=M_{l-1}(x) \frac{1}{b(x)}\left[\begin{array}{cc}
a(x-\omega)-E & -\overline{b(x-\omega)} \\
b(x) & 0
\end{array}\right]
$$


we get

$$
f_{l}(x-\omega)=\frac{a(x-\omega)-E}{b(x)} f_{l-1}(x)-\frac{\overline{b(x)}}{b(x+\omega)} f_{l-2}(x+\omega) .
$$

From this we deduce that

$$
\begin{aligned}
\left|f_{l-1}(x)\right| & =\left|\frac{a(x-\omega)-E}{b(x)}\right|^{-1}\left|f_{l}(x-\omega)+\frac{\overline{b(x)}}{b(x+\omega)} f_{l-2}(x+\omega)\right| \\
& \leq C_{1} \exp \left(\delta-I_{a, E}\right)\left(\exp \left(-C_{1} l^{2}\right)+\exp \left(-\frac{\gamma l}{8}\right)\right)
\end{aligned}
$$

on a subset of $G_{l}$ of measure greater than $1 / 3-\exp \left(-c_{1} \delta+r_{1}\right)-\exp \left(-c_{3} l\right)-$ $\exp \left(-c_{4} l\right)$. By choosing $\delta=\gamma l / 17$ we get

$$
\left|f_{l-1}(x)\right| \leq \exp \left(-\frac{\gamma l}{16}\right)
$$

on a subset of $G_{l}$ of measure greater than $1 / 3-\exp (-c l)$. Now it is easy to see that we have (4.6).

Lemma 4.3. Let $\sigma>0$. There exist constants

$$
l_{0}=l_{0}\left(\|a\|_{\infty}, I_{a, E},\|b\|_{*},|E|, \omega, \gamma, \sigma\right)
$$

and

$$
N_{0}=N_{0}\left(\|a\|_{\infty}, I_{a, E},\|b\|_{*},|E|, \omega, \gamma, \sigma\right)
$$

such that

$$
\operatorname{mes}\left\{x \in \mathbb{T}:\left|f_{l}(x)\right| \leq \exp \left(-N^{\sigma}\right)\right\} \leq \exp \left(-N^{\sigma} l^{-2}\right)
$$

for any $N \geq N_{0}$ and for any $l_{0} \leq l \leq N^{\sigma / 3}$. The same result, but with possibly different $l_{0}$ and $N_{0}$, holds for $f_{l}^{u}$.

Proof. We argue by contradiction. Assume

$$
\operatorname{mes}\left\{x \in \mathbb{T}:\left|f_{l}(x)\right| \leq \exp \left(-N^{\sigma}\right)\right\}>\exp \left(-N^{\sigma} l^{-2}\right)
$$

for some arbitrarily large $l$ and $N$. We have that

$$
\left|f_{l}^{a}(x)\right|=\left|f_{l}(x)\right| \prod_{j=1}^{l}|b(x+j \omega)| \leq \exp \left(-N^{\sigma}\right) C^{l-1} \leq \exp \left(-\frac{N^{\sigma}}{2}\right)
$$

on a set of measure greater than $\exp \left(-N^{\sigma} l^{-2}\right)$. Hence

$$
\operatorname{mes}\left\{x \in \mathbb{T}:\left|f_{l}^{a}(x)\right| \leq \exp \left(-N^{\sigma} / 2\right)\right\}>\exp \left(-N^{\sigma} l^{-2}\right) .
$$


By applying Lemma 4.1 we get that

$$
\left|f_{l}^{a}(x)\right| \leq \exp \left(C_{1} l-\frac{N^{\sigma}}{2 C_{1} \log \left(C_{2} \exp \left(N^{\sigma} l^{-2}\right)\right)}\right) \leq \exp \left(-C l^{2}\right)
$$

for all $x$. Note that the last inequality is equivalent to

$$
\frac{C_{1}}{l}+C \leq \frac{N^{\sigma} l^{-2}}{2 C_{1} \log \left(C_{2} \exp \left(N^{\sigma} l^{-2}\right)\right)}=\frac{N^{\sigma} l^{-2}}{2 C_{1} \log C_{2}+2 C_{1} N^{\sigma} l^{-2}}
$$

which clearly holds with $C=1 /\left(4 C_{1}\right)$ for large $l$ and $N$, since $N^{\sigma} l^{-2} \geq N^{\sigma / 3}$. We now have that

$$
\left|f_{l}(x)\right| \leq \exp \left((l-1)(1-D)-C^{\prime} l^{2}\right) \leq \exp \left(-C l^{2}\right)
$$

for all $x$ except for a set of measure less than $\exp \left(-c_{1}(l-1)+r_{l-1}\right)<\exp (-c l)$. The contradiction follows in the same way as in the previous lemma.

To get the result for $f_{l}^{u}$ one can argue by contradiction. Using

$$
\left|f_{l}^{a}(x)\right|=\left|f_{l}^{u}(x)\right| \prod_{j=0}^{n-1}|b(x+j \omega) b(x+(j+1) \omega)|^{1 / 2}
$$

one can get that $\left|f_{l}^{a}(x)\right| \leq \exp \left(-C l^{2}\right)$ for all $x$ and this gives the same contradiction as before.

We recall for convenience some facts about stability of contracting and expanding directions of unimodular matrices. It follows from the polar decomposition that if $A \in \mathrm{SL}(2, \mathbb{C})$ then there exist unit vectors $u_{A}^{+} \perp u_{A}^{-}$and $v_{A}^{+} \perp v_{A}^{-}$such that $A u_{A}^{+}=\|A\| v_{A}^{+}$and $A u_{A}^{-}=\|A\|^{-1} v_{A}^{-}$.

Lemma 4.4 ([5], Lemma 2.5). For any $A, B \in S L(2, \mathbb{C})$ we have

$$
\begin{gathered}
\left|B u_{A B}^{-} \wedge u_{A}^{-}\right| \leq\|A\|^{-2}\|B\|, \\
\left|u_{B A}^{-} \wedge u_{A}^{-}\right| \leq\|A\|^{-2}\|B\|^{2}, \\
\left|v_{A B}^{+} \wedge v_{A}^{+}\right| \leq\|A\|^{-2}\|B\|^{2}, \\
\left|v_{B A}^{+} \wedge B v_{A}^{+}\right| \leq\|A\|^{-2}\|B\| .
\end{gathered}
$$

We will need the following estimate (cf. [5], eq. (2.35)) in the proof of Lemma 4.6.

Lemma 4.5. If $A \in S L(2, \mathbb{C})$ and $w_{1}, w_{2}$, and $w_{3}$ are unit vectors in the plane then

and

$$
\left|w_{1} \wedge A w_{2}\right| \leq\left|w_{1} \wedge A w_{3}\right|+\sqrt{2}\left\|A^{-1}\right\|\left|w_{2} \wedge w_{3}\right|
$$

$$
\left|w_{1} \wedge A w_{2}\right| \leq\left|w_{3} \wedge A w_{2}\right|+\sqrt{2}\|A\|\left|w_{1} \wedge w_{3}\right|
$$


Proof. Since $A$ preserves area we have

$$
\begin{aligned}
\left|w_{1} \wedge A w_{2}\right| & =\left|A^{-1} w_{1} \wedge w_{2}\right| \\
& \leq\left|A^{-1} w_{1} \wedge w_{3}\right|+\min \left|A^{-1} w_{1} \wedge\left(w_{2} \pm w_{3}\right)\right| \\
& \leq\left|w_{1} \wedge A w_{3}\right|+\left\|A^{-1} w_{1}\right\| \min \left\|w_{2} \pm w_{3}\right\| \\
& \leq\left|w_{1} \wedge A w_{3}\right|+\left\|A^{-1}\right\| \sqrt{2}\left|w_{2} \wedge w_{3}\right|
\end{aligned}
$$

The second inequality follows from the first one.

Let $\mathcal{G}_{N}$ be the set of points $x \in \mathbb{T}$ such that for any $1 \leq j \leq N$ and any $|l| \leq 2 N$ we have

$$
\begin{array}{r}
\left|\log \left\|M_{j}^{u}(x+l \omega)\right\|-j L\right| \leq N^{\sigma}, \\
\log \left\|M_{j}^{u}(x+l \omega)^{-1}\right\| \leq N^{\sigma},
\end{array}
$$

and

$$
|\log | b(x+j \omega)|-D| \leq N^{\sigma}
$$

From Corollary 3.11, Corollary 3.2. and Theorem 2.1 we have that

$$
\begin{aligned}
& \operatorname{mes}\left(\mathbb{T} \backslash \mathscr{G}_{N}\right) \\
& \quad \leq(4 N+1) N \exp \left(-c_{1} N^{\sigma}+r_{N}\right)+(4 N+1) N \exp \left(-c_{2} N^{\sigma}\right) \\
& \quad+N \exp \left(-c_{3} N^{\sigma}+r_{1}^{\prime}\right) \\
& \quad \leq \exp \left(-c N^{\sigma}\right)
\end{aligned}
$$

for $N$ large enough. The choice of $\mathscr{E}_{N}$ is such that all the estimates in the next lemma hold on this set.

Lemma 4.6. Let $0<\sigma<1$. There exist constants

$$
l_{0}=l_{0}\left(\|a\|_{\infty}, I_{a, E},\|b\|_{*},|E|, \omega, \gamma, \sigma\right)
$$

and

$$
N_{0}=N_{0}\left(\|a\|_{\infty}, I_{a, E},\|b\|_{*},|E|, \omega, \gamma, \sigma\right)
$$

such that

$$
\begin{aligned}
& \operatorname{mes}\left\{x \in \mathbb{T}:\left|f_{N}^{u}(x)\right|+\left|f_{N}^{u}\left(x+j_{1} \omega\right)\right|+\left|f_{N}^{u}\left(x+j_{2} \omega\right)\right|\right. \\
& \left.\quad \leq \exp \left(\mathrm{NL}_{N}-100 N^{\sigma}\right)\right\} \\
& \leq \exp \left(-N^{\sigma / 2}\right)
\end{aligned}
$$

for any $l_{0} \leq j_{1} \leq j_{1}+l_{0} \leq j_{2} \leq N^{\sigma / 8}$ and $N \geq N_{0}$ 
Proof. Let $\left\{e_{1}, e_{2}\right\}$ be the standard basis of $\mathbb{R}^{2}$. By (2.6) we have

$$
\begin{aligned}
f_{N}^{u}(x)= & M_{N}^{u}(x) e_{1} \wedge e_{2} \\
= & \left(M_{N}^{u}(x)\left[\left(u_{N}^{+}(x) \cdot e_{1}\right) u_{N}^{+}(x)+\left(u_{N}^{-}(x) \cdot e_{1}\right) u_{N}^{-}(x)\right]\right) \wedge e_{2} \\
= & \left(u_{N}^{+}(x) \cdot e_{1}\right)\left\|M_{N}^{u}(x)\right\| v_{N}^{+}(x) \wedge e_{2} \\
& \quad+\left(u_{N}^{-}(x) \cdot e_{1}\right)\left\|M_{N}^{u}(x)\right\|^{-1} v_{N}^{-}(x) \wedge e_{2} .
\end{aligned}
$$

If $\left|f_{N}^{u}(x)\right| \leq \exp \left(\mathrm{NL}_{N}-100 N^{\sigma}\right)$ then

$$
\begin{aligned}
& \left\|M_{N}^{u}(x)\right\|\left|u _ { N } ^ { + } ( x ) \cdot e _ { 1 } \left\|v_{N}^{+}(x) \wedge e_{2}\left|-\left\|M_{N}^{u}(x)\right\|^{-1}\right| u_{N}^{-}(x) \cdot e_{1}|| v_{N}^{-}(x) \wedge e_{2} \mid\right.\right. \\
& \quad \leq \exp \left(\mathrm{NL}_{N}-100 N^{\sigma}\right) .
\end{aligned}
$$

From the above and the fact that $u_{N}^{+}(x) \cdot e_{1}=u_{N}^{-}(x) \wedge e_{1}\left(\right.$ recall that $\left.u_{N}^{+} \perp u_{N}^{-}\right)$ one gets that on $\mathscr{E}_{N}$ we have

$$
\begin{aligned}
\left|u_{N}^{-}(x) \wedge e_{1}\right|\left|v_{N}^{+}(x) \wedge e_{2}\right| & \leq \exp \left(N\left(L_{N}-L\right)-99 N^{\sigma}\right)+\exp \left(2 N^{\sigma}-2 \mathrm{NL}\right) \\
& \leq \exp \left(-90 N^{\sigma}\right)
\end{aligned}
$$

and hence $\left|u_{N}^{-}(x) \wedge e_{1}\right| \leq \exp \left(-40 N^{\sigma}\right)$ or $\left|v_{N}^{+}(x) \wedge e_{2}\right| \leq \exp \left(-40 N^{\sigma}\right)$.

Suppose (4.7) fails. Then

$$
\begin{gathered}
\operatorname{mes}\left\{x \in \mathcal{E}_{N}:\left|f_{N}^{u}(x)\right|+\left|f_{N}^{u}\left(x+j_{1} \omega\right)\right|+\left|f_{N}^{u}\left(x+j_{2} \omega\right)\right|\right. \\
\left.\leq \exp \left(\mathrm{NL}_{N}-100 N^{\sigma}\right)\right\} \\
>\exp \left(-N^{\sigma / 2}\right)-\exp \left(-c_{1} N^{\sigma}\right)>\exp \left(-c N^{\sigma / 2}\right) .
\end{gathered}
$$

Let $x$ be in the above set. By the preliminary discussion, either

$$
\left|u_{N}^{-}(x) \wedge e_{1}\right| \leq \exp \left(-40 N^{\sigma}\right)
$$

or

$$
\left|v_{N}^{+}(x) \wedge e_{2}\right| \leq \exp \left(-40 N^{\sigma}\right)
$$

has to hold for two of the points $x, x+j_{1} \omega, x+j_{2} \omega$.

We first assume that

$\left|u_{N}^{-}\left(x+j_{1} \omega\right) \wedge e_{1}\right| \leq \exp \left(-40 N^{\sigma}\right)$ and $\left|u_{N}^{-}\left(x+j_{2} \omega\right) \wedge e_{1}\right| \leq \exp \left(-40 N^{\sigma}\right)$.

We now compare $M_{j_{2}-j_{1}}^{u}\left(x+j_{1} \omega\right) u_{N}^{-}\left(x+j_{1} \omega\right)$ and $u_{N}^{-}\left(x+j_{2} \omega\right)$. It follows from Lemma 4.5 that

$$
\begin{aligned}
& \left|u_{N}^{-}\left(x+j_{2} \omega\right) \wedge M_{j_{2}-j_{1}}^{u}\left(x+j_{1} \omega\right) u_{N}^{-}\left(x+j_{1} \omega\right)\right| \\
& \quad \leq\left|u_{N}^{-}\left(x+j_{2} \omega\right) \wedge M_{j_{2}-j_{1}}^{u}\left(x+j_{1} \omega\right) u_{N+j_{2}-j_{1}}^{-}\left(x+j_{1} \omega\right)\right| \\
& \quad+C\left\|M_{j_{2}-j_{1}}^{u}\left(x+j_{1} \omega\right)^{-1}\right\|\left|u_{N+j_{2}-j_{1}}^{-}\left(x+j_{1} \omega\right) \wedge u_{N}^{-}\left(x+j_{1} \omega\right)\right| .
\end{aligned}
$$


Applying Lemma 4.4 with $A=M_{N}^{u}\left(x+j_{2} \omega\right)$ and $B=M_{j_{2}-j_{1}}^{u}\left(x+j_{1} \omega\right)$ for the first term, and $A=M_{N}^{u}\left(x+j_{1} \omega\right)$ and $B=M_{j_{2}-j_{1}}^{u}\left(x+\left(N+j_{1}\right) \omega\right)$ for the second term, yields

$$
\begin{aligned}
& \left|u_{N}^{-}\left(x+j_{2} \omega\right) \wedge M_{j_{2}-j_{1}}^{u}\left(x+j_{1} \omega\right) u_{N}^{-}\left(x+j_{1} \omega\right)\right| \\
& \quad \leq\left\|M_{N}^{u}\left(x+j_{2} \omega\right)\right\|^{-2}\left\|M_{j_{2}-j_{1}}^{u}\left(x+j_{1} \omega\right)\right\| \\
& \quad+C\left\|M_{j_{2}-j_{1}}^{u}\left(x+j_{1} \omega\right)^{-1}\right\|\left\|M_{N}^{u}\left(x+j_{1} \omega\right)\right\|^{-2}\left\|M_{j_{2}-j_{1}}^{u}\left(x+\left(N+j_{1}\right) \omega\right)\right\|^{2} \\
& \quad \leq \exp \left(\left(-2 N+j_{2}-j_{1}\right) L+3 N^{\sigma}\right)+C \exp \left(\left(-2 N+2\left(j_{2}-j_{1}\right)\right) L+5 N^{\sigma}\right) \\
& \quad \leq \exp (-\mathrm{NL})
\end{aligned}
$$

for $x \in \mathcal{S}_{N}$. Using Lemma 4.5, (4.8), and (4.9) we get

$$
\begin{aligned}
\mid e_{1} \wedge & M_{j_{2}-j_{1}}^{u}\left(x+j_{1} \omega\right) e_{1} \mid \\
\leq & \left|e_{1} \wedge M_{j_{2}-j_{1}}^{u}\left(x+j_{1} \omega\right) u_{N}^{-}\left(x+j_{1} \omega\right)\right| \\
& \quad+C\left\|M_{j_{2}-j_{1}}^{u}\left(x+j_{1} \omega\right)^{-1}\right\|\left|e_{1} \wedge u_{N}^{-}\left(x+j_{1} \omega\right)\right| \\
\leq & \left|u_{N}^{-}\left(x+j_{2} \omega\right) \wedge M_{j_{2}-j_{1}}^{u}\left(x+j_{1} \omega\right) u_{N}^{-}\left(x+j_{1} \omega\right)\right| \\
& \quad+C\left\|M_{j_{2}-j_{1}}^{u}\left(x+j_{1} \omega\right)\right\|\left|e_{1} \wedge u_{N}^{-}\left(x+j_{2} \omega\right)\right| \\
& \quad+C\left\|M_{j_{2}-j_{1}}^{u}\left(x+j_{1} \omega\right)^{-1}\right\|\left|e_{1} \wedge u_{N}^{-}\left(x+j_{1} \omega\right)\right| \\
\leq & \exp (-\mathrm{NL})+C \exp \left(\left(j_{2}-j_{1}\right) L-39 N^{\sigma}\right)+C \exp \left(-39 N^{\sigma}\right) \\
\leq & \exp \left(-30 N^{\sigma}\right) .
\end{aligned}
$$

On the other hand by (2.6) we have

$$
\left|e_{1} \wedge M_{j_{2}-j_{1}}^{u}\left(x+j_{1} \omega\right) e_{1}\right|=\left|\frac{b\left(x+j_{2} \omega\right)}{b\left(x+\left(j_{2}-1\right) \omega\right)}\right|^{1 / 2}\left|f_{j_{2}-j_{1}-1}^{u}\left(x+j_{1} \omega\right)\right|,
$$

so

$$
\left|f_{j_{2}-j_{1}-1}^{u}\left(x+j_{1} \omega\right)\right| \leq C \exp \left(\frac{1}{2}\left(N^{\sigma}-D\right)-30 N^{\sigma}\right) \leq \exp \left(-20 N^{\sigma}\right) .
$$

The same type of estimate is obtained if we replace $\left(j_{1}, j_{2}\right)$ in $(4.8)$ with $\left(0, j_{1}\right)$ or $\left(0, j_{2}\right)$.

Now assume that

$$
\left|v_{N}^{+}\left(x+j_{1} \omega\right) \wedge e_{2}\right| \leq \exp \left(-40 N^{\sigma}\right)
$$

and

$$
\left|v_{N}^{+}\left(x+j_{2} \omega\right) \wedge e_{2}\right| \leq \exp \left(-40 N^{\sigma}\right) .
$$


Similarly to the previous case (first use Lemma 4.5 and then Lemma 4.4) we have

$$
\begin{aligned}
& \left|v_{N}^{+}\left(x+j_{2} \omega\right) \wedge M_{j_{2}-j_{1}}^{u}\left(x+\left(N+j_{1}\right) \omega\right) v_{N}^{+}\left(x+j_{1} \omega\right)\right| \\
& \leq\left|v_{N+j_{2}-j_{1}}^{+}\left(x+j_{1} \omega\right) \wedge M_{j_{2}-j_{1}}^{u}\left(x+\left(N+j_{1}\right) \omega\right) v_{N}^{+}\left(x+j_{1} \omega\right)\right| \\
& \quad+C\left\|M_{j_{2}-j_{1}}^{u}\left(x+\left(N+j_{1}\right) \omega\right)\right\|\left|v_{N}^{+}\left(x+j_{2} \omega\right) \wedge v_{N+j_{2}-j_{1}}^{+}\left(x+j_{1} \omega\right)\right| \\
& \leq \mid\left\|M_{N}^{u}\left(x+j_{1} \omega\right)\right\|^{-2}\left\|M_{j_{2}-j_{1}}^{u}\left(x+\left(N+j_{1}\right) \omega\right)\right\| \\
& \quad+C\left\|M_{j_{2}-j_{1}}^{u}\left(x+\left(N+j_{1}\right) \omega\right)\right\|\left\|M_{N}^{u}\left(x+j_{2} \omega\right)\right\|^{-2}\left\|M_{j_{2}-j_{1}}^{u}\left(x+j_{1} \omega\right)\right\|^{2} \\
& \quad \leq \exp \left(\left(-2 N+j_{2}-j_{1}\right) L+3 N^{\sigma}\right)+C \exp \left(\left(-2 N+3\left(j_{2}-j_{1}\right)\right) L+5 N^{\sigma}\right) \\
& \leq \exp (-\mathrm{NL})
\end{aligned}
$$

for $x \in \mathscr{E}_{N}$ and

$$
\begin{aligned}
\mid e_{2} \wedge & M_{j_{2}-j_{1}}^{u}\left(x+\left(N+j_{1}\right) \omega\right) e_{2} \mid \\
\leq & \left|e_{2} \wedge M_{j_{2}-j_{1}}^{u}\left(x+\left(N+j_{1}\right) \omega\right) v_{N}^{+}\left(x+j_{1} \omega\right)\right| \\
& +C\left\|M_{j_{2}-j_{1}}^{u}\left(x+\left(N+j_{1}\right) \omega\right)^{-1}\right\|\left|e_{2} \wedge v_{N}^{+}\left(x+j_{1} \omega\right)\right| \\
\leq & \left|v_{N}^{+}\left(x+j_{2} \omega\right) \wedge M_{j_{2}-j_{1}}^{u}\left(x+\left(N+j_{1}\right) \omega\right) v_{N}^{+}\left(x+j_{1} \omega\right)\right| \\
& +C\left\|M_{j_{2}-j_{1}}^{u}\left(x+\left(N+j_{1}\right) \omega\right)\right\|\left|e_{2} \wedge v_{N}^{+}\left(x+j_{2} \omega\right)\right| \\
& +C\left\|M_{j_{2}-j_{1}}^{u}\left(x+\left(N+j_{1}\right) \omega\right)^{-1}\right\|\left|e_{2} \wedge v_{N}^{+}\left(x+j_{1} \omega\right)\right| \\
\leq & \exp (-\mathrm{NL})+C \exp \left(\left(j_{2}-j_{1}\right) L-39 N^{\sigma}\right)+C \exp \left(-39 N^{\sigma}\right) \\
\leq & \exp \left(-30 N^{\sigma}\right) .
\end{aligned}
$$

On the other hand by (2.6) we have

$$
\begin{aligned}
\mid e_{2} & \wedge M_{j_{2}-j_{1}}^{u}\left(x+\left(N+j_{1}\right) \omega\right) e_{2} \mid \\
& =\left|\frac{b\left(x+\left(N+j_{1}\right) \omega\right)}{b\left(x+\left(N+j_{1}+1\right) \omega\right)}\right|^{1 / 2}\left|f_{j_{2}-j_{1}-1}^{u}\left(x+\left(N+j_{1}+1\right) \omega\right)\right|,
\end{aligned}
$$

so

$$
\begin{aligned}
\left|f_{j_{2}-j_{1}-1}^{u}\left(x+\left(N+j_{1}+1\right) \omega\right)\right| & \leq C \exp \left(\frac{1}{2}\left(N^{\sigma}-D\right)-30 N^{\sigma}\right) \\
& \leq \exp \left(-20 N^{\sigma}\right) .
\end{aligned}
$$

In conclusion

$$
\operatorname{mes}\left\{x \in \mathbb{T}:\left|f_{l}^{u}(x)\right| \leq \exp \left(-20 N^{\sigma}\right)\right\}>\exp \left(-c N^{\sigma / 2}\right)
$$

for some choice of $l$ from $j_{1}-1, j_{2}-1, j_{2}-j_{1}-1$. However, this contradicts the 
fact that Lemma 4.3 implies

$$
\begin{aligned}
\operatorname{mes}\left\{x \in \mathbb{T}:\left|f_{l}^{u}(x)\right|\right. & \left.\leq \exp \left(-20 N^{\sigma}\right)\right\} \\
& \leq \operatorname{mes}\left\{x \in \mathbb{T}:\left|f_{l}^{u}(x)\right| \leq \exp \left(-N^{\sigma}\right)\right\} \\
& \leq \exp \left(-N^{\sigma} l^{-2}\right) \leq \exp \left(-N^{3 \sigma / 4}\right)<\exp \left(-c N^{\sigma / 2}\right)
\end{aligned}
$$

(we used $l \leq N^{\sigma / 8}$ ).

Lemma 4.7. There exist constants $\kappa>0$ and

$$
N_{0}=N_{0}\left(\|a\|_{\infty}, I_{a, E},\|b\|_{*},|E|, \omega, \gamma\right)
$$

such that for $N \geq N_{0}$ we have

$$
\int_{\mathbb{T}} \frac{1}{N}\left|f_{N}^{u}(x)\right| d x>L_{N}-N^{-\kappa} .
$$

Proof. Let $\Omega_{N}$ be the set of points $x \in \mathscr{Y}_{N}$ such that

$$
\begin{aligned}
& \min \left\{\left|f_{N}^{u}\left(x+j_{1} \omega\right)\right|+\left|f_{N}^{u}\left(x+j_{2} \omega\right)\right|+\left|f_{N}^{u}\left(x+j_{3} \omega\right)\right|:\right. \\
& \left.0<j_{1}<j_{1}+l_{0} \leq j_{2}<j_{2}+l_{0} \leq j_{3} \leq N^{\sigma / 8}\right\} \\
& >\exp \left(\mathrm{NL}_{N}-100 N^{\sigma}\right),
\end{aligned}
$$

where $l_{0}$ is as in the previous lemma. If $N$ is large enough then

$$
\operatorname{mes}\left(\mathbb{T} \backslash \Omega_{N}\right) \leq N \exp \left(-c_{1} N^{\sigma / 2}\right)<\exp \left(-c N^{\sigma / 2}\right) .
$$

Let $u(x)=\log \left|f_{N}^{u}(x)\right| / N$ and set

$$
M=\left[N^{\sigma / 8} / l_{0}\right] .
$$

For each $x \in \Omega_{N}$ we have that

$$
\left|f_{N}^{u}\left(x+k l_{0} \omega\right)\right|>\exp \left(\mathrm{NL}_{N}-100 N^{\sigma}\right) / 3
$$

for all but at most two $k$ 's, $1 \leq k \leq M$. We have

$$
\begin{aligned}
\langle u\rangle \stackrel{\text { def }}{=} \int_{\mathbb{T}} u(x) d x & \\
= & \frac{1}{M} \sum_{k=1}^{M} \int_{\mathbb{T}} u\left(x+k l_{0} \omega\right) d x \\
\geq & \int_{\Omega_{N}}\left(\frac{M-2}{M}\left(L_{N}-100 N^{\sigma-1}-\frac{\log 3}{N}\right)+\frac{2}{M} \inf _{1 \leq k \leq M} u\left(x+k l_{0} \omega\right)\right) d x \\
& \quad+\frac{1}{M} \sum_{k=1}^{M} \int_{\mathbb{T} \backslash \Omega_{N}} u\left(x+k l_{0} \omega\right) d x .
\end{aligned}
$$


Let $v(x)=\log \left|f_{N}^{a}(x)\right| / N$. We have that

$$
S \stackrel{\text { def }}{=} \sup _{z \in \mathcal{A}_{\rho_{0}^{\prime \prime}}} v(z) \leq \sup _{z \in \mathcal{A}_{\rho_{0}^{\prime \prime}}} \frac{1}{N} \log \left\|M_{N}^{a}(z)\right\|<\infty .
$$

Let

$$
v(z)=\int_{\mathscr{A}_{\rho_{0}^{\prime}}^{\prime}} \log |z-\zeta| d \mu(\zeta)+h(z)
$$

be the Riesz representation on $\mathcal{A}_{\rho_{0}^{\prime}}$. Applying [5], Lemma 2.2, (see the proof of Lemma 3.4) we get that

$$
\mu\left(\mathcal{A}_{\rho_{0}}\right)+\|h\|_{L^{\infty}\left(\mathcal{A}_{\rho_{0}}\right)} \leq C\left(2 S-\sup _{\mathbb{T}} v\right) \leq C(2 S-\langle v\rangle) .
$$

Note that $\langle v\rangle$ is finite by subharmonicity. Since $\langle v\rangle=\langle u\rangle+D$, it follows that $\langle u\rangle$ is also finite. Using Cartan's estimate (see [4], Lemma 2.2) we get that for any small $\varepsilon>0$ we have

$$
\inf _{1 \leq k \leq M} v\left(x+k l_{0} \omega\right) \geq-C(2 S-\langle v\rangle) N^{\varepsilon}
$$

up to a set not exceeding $C M \exp \left(-N^{\varepsilon}\right)$ in measure. Since

$$
u(x)=v(x)-\frac{1}{2 N}\left(S_{N}(x)+S_{N}(x+\omega)\right)
$$

we can use (4.12) and Theorem 2.1 to conclude that

$$
\inf _{1 \leq k \leq M} u\left(x+k l_{0} \omega\right)>-C(2 S-\langle u\rangle-D) N^{\varepsilon}-D-N^{\varepsilon}>\left(C\langle u\rangle-C^{\prime}\right) N^{\varepsilon}
$$

up to a set $\mathscr{B}_{N}$ not exceeding $\exp \left(-c N^{\varepsilon}\right)$ in measure. Therefore

$$
\begin{aligned}
& \int_{\Omega_{N}} \inf _{1 \leq k \leq M} u\left(x+k l_{0} \omega\right) d x \\
& >\left(C\langle u\rangle-C^{\prime}\right) N^{\varepsilon}+\int_{\Omega_{N} \cap \mathcal{B}_{N}} \inf _{1 \leq k \leq M} u\left(x+k l_{0} \omega\right) \\
& >\left(C\langle u\rangle-C^{\prime}\right) N^{\varepsilon}-\sum_{k=1}^{M} \int_{\Omega_{N} \cap \mathcal{B}_{N}}\left|u\left(x+k l_{0} \omega\right)\right| d x .
\end{aligned}
$$

Now (4.10) becomes

$$
\begin{gathered}
\langle u\rangle \geq\left(1-\frac{2}{M}\right)\left(L_{N}-100 N^{\sigma-1}-\frac{\log 3}{N}\right)+\frac{\left(C\langle u\rangle-C^{\prime}\right) N^{\varepsilon}}{M} \\
-\frac{2}{M} \sum_{k=1}^{M} \int_{\Omega_{N}^{c} \cup B_{N}}\left|u\left(x+k l_{0} \omega\right)\right| .
\end{gathered}
$$


Using Lemma 4.3 (with $\sigma=3$ ) and reasoning as in the proof of Lemma 3.8 we get that $\|u\|_{L^{2}(\mathbb{T})} \leq C N^{3}$ and consequently

$$
\int_{\Omega_{N}^{c} \cup \mathcal{B}_{N}}\left|u\left(x+k l_{0} \omega\right)\right| d x \leq\left(\operatorname{mes}\left\{\Omega_{N}^{c} \cup \mathcal{B}_{N}\right\}\right)^{1 / 2}\|u\|_{L^{2}(\mathbb{\Psi})} \leq C N^{3} \exp \left(-c N^{\varepsilon}\right) .
$$

Now it is straightforward to reach the conclusion.

\section{Corollary 4.8. Let}

$$
\frac{1}{n} \log \left|f_{n}^{a}(z)\right|=\int_{\mathcal{A}_{\rho_{0}^{\prime}}} \log |z-\zeta| d \mu_{n}(\zeta)+h_{n}(z)
$$

be the Riesz representation on $\mathcal{A}_{\rho_{0}^{\prime}}$. There exists a constant

$$
C_{0}=C_{0}\left(\|a\|_{\infty}, I_{a, E},\|b\|_{*},|E|, \omega, \gamma, \rho_{0}, \rho_{0}^{\prime}, \rho_{0}^{\prime \prime}\right)
$$

such that

$$
\mu_{n}\left(\mathcal{A}_{\rho_{0}}\right)+\left\|h_{n}\right\|_{L^{\infty}\left(\mathcal{A}_{\rho_{0}}\right)} \leq C_{0} .
$$

Proof. It suffices to obtain the bound for large $n$. The bound follows from (4.11) and the previous lemma.

Lemma 4.9. There exist constants $\sigma_{0}>0$,

$$
c_{0}=c_{0}\left(I_{a, E},\|b\|_{*},|E|, \omega, \gamma\right)
$$

and

$$
C_{0}=C_{0}\left(I_{a, E},\|b\|_{*},|E|, \omega, \gamma\right)
$$

such that for every integer $n$ and any $\delta>0$ we have

$$
\operatorname{mes}\left\{x \in \mathbb{T}:|\log | f_{n}^{a}(x)\left|-\left\langle\log \left|f_{n}^{a}\right|\right\rangle\right|>n \delta\right\} \leq C_{0} \exp \left(-c_{0} \delta n^{\sigma_{0}}\right) .
$$

The same estimate with possibly different $c_{0}$ and $C_{0}$ holds for $f_{n}^{u}$.

Proof. It is enough to establish the estimate for $n$ large enough. Let $u(x)=$ $\log \left|f_{n}^{u}(x)\right| / n$ and $v(x)=\log \left|f_{n}^{a}(x)\right| / n$. By the previous lemma (recall that $\langle v\rangle=\langle u\rangle+D$ ) and Proposition 3.14 we have that there exists a small $\kappa>0$ such that

$$
\left\{\begin{array}{l}
\langle v\rangle \geq L_{n}^{a}-n^{-\kappa} \\
\sup _{\mathbb{T}} v \leq L_{n}^{a}+n^{-\kappa} .
\end{array}\right.
$$

This implies that

$$
\|v-\langle v\rangle\|_{L^{1}(\mathbb{T})} \leq C n^{-\kappa}
$$


and hence by [1], Lemma 2.3, we have

$$
\|v\|_{\mathrm{BMO}(\mathbb{T})}=\|v-\langle v\rangle\|_{\mathrm{BMO}(\mathbb{T})} \leq C\|v-\langle v\rangle\|_{L^{1}(\mathbb{T})}^{1 / 2} \leq C n^{-\kappa / 2} .
$$

As in the proof of [5], Proposition 2.11, we note that in order to get the conclusion of [1], Lemma 2.3, we just need the bounds on the Riesz representation of $v$. By the John-Nirenberg inequality we get

$$
\operatorname{mes}\{x \in \mathbb{T}:|v(x)-\langle v\rangle|>\delta\} \leq C \exp \left(-c \delta n^{\kappa / 2}\right) .
$$

Using (4.13) we have

$$
\begin{aligned}
\operatorname{mes} & \{x \in \mathbb{T}:|u(x)-\langle u\rangle|>\delta\} \\
\leq & \operatorname{mes}\left\{x \in \mathbb{T}:|v(x)-\langle v\rangle|>\frac{\delta}{2}\right\} \\
& \quad+\operatorname{mes}\left\{x \in \mathbb{T}:\left|\frac{1}{2 n}\left(S_{n}(x)+S_{n}(x+\omega)\right)-D\right|>\frac{\delta}{2}\right\} \\
\leq & C \exp \left(-c \delta n^{\kappa / 2} / 2\right)+2 \exp \left(-c^{\prime} \delta n / 2+r_{n}\right) \\
\leq & C^{\prime} \exp \left(-c^{\prime \prime} \delta n^{\kappa / 2} / 2\right) .
\end{aligned}
$$

This concludes the proof.

Next we will use the Avalanche Principle to refine the previous estimate.

Proposition 4.10. There exist constants

$$
\begin{aligned}
c_{0} & =c_{0}\left(\|a\|_{\infty}, I_{a, E},\|b\|_{*},|E|, \omega, \gamma\right), \\
C_{0} & =C_{0}(\omega)>\alpha+2,
\end{aligned}
$$

and

$$
C_{1}=C_{1}\left(\|a\|_{\infty}, I_{a, E},\|b\|_{*},|E|, \omega, \gamma\right)
$$

such that for every integer $n>1$ and any $\delta>0$ we have

$$
\begin{aligned}
& \operatorname{mes}\left\{x \in \mathbb{T}:|\log | f_{n}^{a}(x)\left|-\left\langle\log \left|f_{n}^{a}\right|\right\rangle\right|>n \delta\right\} \\
& \quad \leq C_{1} \exp \left(-c_{0} \delta n(\log n)^{-C_{0}}\right) .
\end{aligned}
$$

Proof. It is enough to establish the estimate for $n$ large enough. We have that

$$
\left[\begin{array}{cc}
f_{n}^{u}(x) & 0 \\
0 & 0
\end{array}\right]=\left[\begin{array}{cc}
-1 & 0 \\
0 & 0
\end{array}\right] M_{n}^{u}(x)\left[\begin{array}{cc}
-1 & 0 \\
0 & 0
\end{array}\right] \stackrel{\text { def }}{=} \mathcal{M}_{n}^{u}(x) .
$$

We define $\mathcal{M}_{n}^{a}$ analogously. We obviously have that $\left|f_{n}^{a}(x)\right|=\left\|\mathcal{M}_{n}^{a}(x)\right\|$. 
Let $l=\left[(\log n)^{2 / \sigma_{0}}\right]$ with $\sigma_{0}$ as in Lemma 4.9. Let $n=l+(m-2) l+l^{\prime}$ with $2 l \leq l^{\prime} \leq 3 l$. We want to apply the Avalanche Principle to $\mathcal{M}_{n}^{u}(x)=\prod_{j=m}^{1} A_{j}^{u}(x)$ where $A_{j}^{u}(x)=M_{l}^{u}(x+(j-1) l \omega), j=2, \ldots, m-1$,

$$
A_{1}^{u}(x)=M_{l}^{u}(x)\left[\begin{array}{cc}
-1 & 0 \\
0 & 0
\end{array}\right]=\left[\begin{array}{cc}
f_{l}^{u}(x) & 0 \\
\star & 0
\end{array}\right],
$$

and

$$
A_{m}^{u}(x)=\left[\begin{array}{cc}
-1 & 0 \\
0 & 0
\end{array}\right] M_{l^{\prime}}^{u}(x)=\left[\begin{array}{cc}
f_{l^{\prime}}^{u}(x) & \star \\
0 & 0
\end{array}\right] .
$$

We define the matrices $A_{j}^{a}$ analogously. We clearly have that

$$
\log \left|f_{l}^{u}(x)\right| \leq \log \left\|A_{1}^{u}(x)\right\| \leq \log \left\|M_{l}^{u}(x)\right\|,
$$

and an analogous estimate for $\log \left\|A_{m}^{u}\right\|$. Now it follows from Corollary 3.11, Lemma 4.9, and Lemma 4.7 that the hypotheses of Lemma 3.7 are satisfied and hence

$$
\log \left\|\mathcal{M}_{n}^{a}(x)\right\|+\sum_{j=2}^{m-1} \log \left\|A_{j}^{a}(x)\right\|-\sum_{j=1}^{m-1} \log \left\|A_{j+1}^{a}(x) A_{j}^{a}(x)\right\|=O\left(\frac{1}{l}\right)
$$

up to a set of measure less than $3 n \exp \left(-c l^{\sigma_{0}}\right)<\exp \left(-c^{\prime}(\log n)^{2}\right)$. Note that, as before, we checked the conditions of the Avalanche Principle for $\mathcal{M}_{n}^{u}$, but we wrote the conclusion for $\mathcal{M}_{n}^{a}$. By letting

$$
u_{0}(x)=\log \left\|A_{m}^{a}(x) A_{m-1}^{a}(x)\right\|+\log \left\|A_{2}^{a}(x) A_{1}^{a}(x)\right\|
$$

we rewrite the previous relation as

$$
\begin{aligned}
\log \left\|\mathcal{M}_{n}^{a}(x)\right\|+\sum_{j=2}^{m-1} \log \left\|M_{l}^{a}(x+(j-1) l \omega)\right\| \\
-\sum_{j=2}^{m-2} \log \left\|M_{2 l}^{a}(x+(j-1) l \omega)\right\|-u_{0}(x) \\
=O\left(\frac{1}{l}\right) .
\end{aligned}
$$

Note that

$$
\begin{gathered}
\log \left\|f_{l+l^{\prime}}^{a}(x+(m-2) l \omega)\right\|+\log \left\|f_{2 l}^{a}(x)\right\| \leq u_{0}(x) \\
\leq \log \left\|M_{l+l^{\prime}}^{a}(x+(m-2) l \omega)\right\|+\log \left\|M_{2 l}^{a}(x)\right\| .
\end{gathered}
$$


We apply the Avalanche Principle $l-1$ more times. At each step we decrease the length of $A_{m}$ by one and increase the length of $A_{1}$ by one. Adding the resulting estimates and dividing by $l$ yields

$$
\begin{aligned}
\log \left\|\mathcal{M}_{n}^{a}(x)\right\| & +\sum_{j=l}^{(m-1) l-1} \frac{1}{l} \log \left\|M_{l}^{a}(x+j \omega)\right\| \\
& -\sum_{j=l}^{(m-2) l-1} \frac{1}{l} \log \left\|M_{2 l}^{a}(x+j \omega)\right\|-\sum_{k=0}^{l-1} \frac{1}{l} u_{k}(x) \\
= & O\left(\frac{1}{l}\right)
\end{aligned}
$$

up to a set of measure less than $l \exp \left(-c(\log n)^{2}\right)<\exp \left(-c^{\prime}(\log n)^{2}\right)$. The functions $u_{k}, k=1, \ldots, l-1$ are defined analogously to $u_{0}$ and satisfy estimates analogous to (4.14). Based on these estimates it is straightforward to conclude (see Lemma 3.4 and (4.11)) that there is an uniform bound for the Riesz representations of $u_{k} / l$, $k=1, \ldots, l-1$. Hence we can use Theorem 2.1 to get

$$
\sum_{k=0}^{l-1} \frac{1}{l} u_{k}(x)-\sum_{k=0}^{l-1} \frac{1}{l}\left\langle u_{k}\right\rangle=O\left(l(\log n)^{2}\right)=O\left((\log n)^{2+2 / \sigma_{0}}\right)
$$

up to a set of measure less than $l \exp \left(-c(\log n)^{2}\right)<\exp \left(-c^{\prime}(\log n)^{2}\right)$. On the other hand, using Theorem 3.10 we have

$$
\begin{aligned}
\sum_{j=l}^{(m-1) l-1} \frac{1}{l} \log \left\|M_{l}^{a}(x+j \omega)\right\| & -\sum_{j=l}^{(m-2) l-1} \frac{1}{l} \log \left\|M_{2 l}^{a}(x+j \omega)\right\| \\
& =(m-2) l L_{l}^{a}-(m-3) l L_{2 l}^{a}+O\left((\log n)^{p}\right)
\end{aligned}
$$

up to a set of measure less than $\exp \left(-c(\log n)^{p}\right)$. We can now conclude from (4.15) that

$$
\log \left|f_{n}^{a}(x)\right|+(m-2) l L_{l}^{a}-(m-3) l L_{2 l}^{a}-\sum_{k=0}^{l-1} \frac{1}{l}\left\langle u_{k}\right\rangle=O\left((\log n)^{C_{2}}\right)
$$

up to a set of measure less than $\exp \left(-c(\log n)^{2}\right)$, where $C_{2}=\max \left\{p, 2+2 / \sigma_{0}\right\}$. Integrating the above relation and then subtracting it, yields

$$
|\log | f_{n}^{a}(x)\left|-\left\langle\log \left|f_{n}^{a}\right|\right\rangle\right| \leq C(\log n)^{C_{2}}
$$

up to a set of measure less than $\exp \left(-c(\log n)^{2}\right)$. Note that the exceptional set was handled by using the fact that $\left\|\log \left|f_{n}^{a}\right|\right\|_{L^{2}(\mathbb{T})} \leq C n$. This follows from

$$
\left\|\log \left|f_{n}^{a}\right|-\left\langle\log \left|f_{n}^{a}\right|\right\rangle\right\|_{L^{2}(\mathbb{T})} \leq C n
$$


and $\left|\left\langle\log \left|f_{n}^{a}\right|\right\rangle\right| \leq C n$. The first estimate is an immediate consequence of Lemma 4.9 and Lemma 3.8. The second estimate can be deduced from Lemma 4.7.

Let $\mathscr{B}$ be the exceptional set for (4.16). Let

$$
\log \left|f_{n}^{a}\right|-\left\langle\log \left|f_{n}^{a}\right|\right\rangle=u_{0}+u_{1}
$$

where $u_{0}=0$ on $\mathscr{B}$ and $u_{1}=0$ on $\mathbb{} \backslash \mathcal{B}$. By (4.16) and (4.17) we have that $\left\|u_{0}-\left\langle u_{0}\right\rangle\right\|_{L^{\infty}(\mathbb{T})} \leq C(\log n)^{C_{2}}$ and

$$
\left\|u_{1}\right\|_{L^{2}(\mathbb{T})} \leq C n \sqrt{\operatorname{mes}(\mathscr{B})} \leq \exp \left(-c(\log n)^{2}\right) .
$$

Applying [1], Lemma 2.3, we have

$$
\left\|\log \left|f_{n}^{a}\right|\right\|_{\mathrm{BMO}(\mathbb{T})} \leq C\left((\log n)^{C_{2}+2}+\sqrt{n \exp \left(-c(\log n)^{2}\right)}\right) \leq C^{\prime}(\log n)^{C_{0}} .
$$

The conclusion follows from the John-Nirenberg inequality.

Lemma 4.11. There exists a constant

$$
C_{0}=C_{0}\left(\|a\|_{\infty}, I_{a, E},\|b\|_{*},|E|, \omega, \gamma\right)
$$

such that

$$
\left|\left\langle\log \left|f_{n}^{a}\right|\right\rangle-n L_{n}^{a}\right| \leq C_{0}
$$

for all integers.

Proof. Subtracting the Avalanche Principle expansions for $\mathcal{M}_{n}^{a}$ and $M_{n}^{a}$ at scale $l \approx$ $(\log n)^{A}$ and then integrating, yields

$$
\left|\left\langle\log \left|f_{n}^{a}\right|\right\rangle-n L_{n}^{a}\right| \leq C R\left(4(\log n)^{A}\right)+O\left(\frac{1}{l}\right)
$$

where

$$
R(n)=\sup _{n / 2 \leq m \leq n}\left|\left\langle\log \left|f_{m}^{a}\right|\right\rangle-m L_{m}^{a}\right|
$$

Iterating this estimate yields the desired conclusion (cf. [5], Lemma 3.5).

We now prepare to prove the estimate on the number of eigenvalues. Fix $E_{0} \in \mathbb{R}$ such that $L\left(E_{0}\right) \geq \gamma>0$. As a consequence of Corollary 3.17 and Lemma 3.9 it follows that there exists a disk $\mathscr{D}$ around $E_{0}$ such that $L(E) \geq \gamma / 2$ on $I$. In what follows we also fix $\mathscr{D}$. Note that the existence of the disk $\mathcal{D}$ would follow from the continuity of the Lyapunov exponent, which is known from [8]. However, we also need the information on the modulus of continuity provided by Corollary 3.17. This information follows from the Hölder continuity of the Lyapunov exponent proved in [10], but we use Corollary 3.17 in order to keep the paper self-contained. The following deviations estimate in $E$ will be needed in the proof of the estimate. 
Lemma 4.12. Let $C_{0}$ be as in Proposition 4.10. There exist constants

$$
c_{0}=c_{0}\left(\|a\|_{\infty}, I_{a, E},\|b\|_{*},|E|, \omega, \gamma\right)
$$

and

$$
C_{1}=C_{1}\left(\|a\|_{\infty}, I_{a, E},\|b\|_{*},|E|, \omega, \gamma\right)
$$

such that for every integer $n>1$ and every $\delta \geq \delta_{0}$ there exists a set $\mathscr{B}_{n, \delta} \subset \mathbb{T}$ with mes $\mathscr{B}_{n, \delta}<C_{1} \exp \left(-c_{0} \delta(\log n)^{-C_{0}}\right)$, such that for each $x \in \mathbb{T} \backslash \mathscr{B}_{n, \delta}$ there exists $\varepsilon_{n, \delta, x} \subset \mathcal{D}$, with mes $\varepsilon_{n, \delta, x}<C_{1} \exp \left(-c_{0} \delta(\log n)^{-C_{0}}\right)$, such that

$$
|\log | f_{n}^{a}(x, E)\left|-n L_{n}^{a}(E)\right| \leq \delta,
$$

for any $E \in \mathscr{D} \backslash \varepsilon_{n, \delta, x}$.

Proof. From Proposition 4.10 and Lemma 4.11 it follows that (4.18) holds for $\delta \geq$ $\delta_{0}$, and $(x, E) \in \mathbb{T} \times \mathbb{D}$ except for a set of measure $C \exp \left(-c \delta /(\log n)^{C_{0}}\right)$. The conclusion follows by Fubini's Theorem and Chebyshev's inequality.

Theorem 4.13. Let $C_{0}=C_{0}(\omega)$ be as in Proposition 4.10. There exist constants

$$
\begin{aligned}
& C_{1}=C_{1}\left(\|a\|_{\infty},\|b\|_{*},\left|E_{0}\right|, \omega, \gamma\right), \\
& C_{2}=C_{2}\left(\|a\|_{\infty}, I_{a, D},\|b\|_{*},\left|E_{0}\right|, \omega, \gamma\right),
\end{aligned}
$$

and

$$
n_{0}=n_{0}\left(\|a\|_{\infty}, I_{a, D},\|b\|_{*},\left|E_{0}\right|, \omega, \gamma\right)
$$

such that for any $x_{0} \in \mathbb{T}, E_{0} \in \mathbb{R}$, and $n \geq n_{0}$ one has

$$
\#\left\{E \in \mathbb{R}: f_{n}^{a}\left(x_{0}, E\right)=0,\left|E-E_{0}\right|<n^{-C_{1}}\right\} \leq C_{2}(\log n)^{2 C_{0}}
$$

and

$$
\#\left\{z \in \mathbb{C}: f_{n}^{a}\left(z, E_{0}\right)=0,\left|z-x_{0}\right|<n^{-1}\right\} \leq C_{2}(\log n)^{2 C_{0}} .
$$

Proof. From (4.18) it follows that there exist $x_{1}, E_{1}$ such that

$$
\begin{aligned}
\left|x_{1}-x_{0}\right| & \leq C \exp \left(-c(\log n)^{C_{0}}\right), \\
\left|E_{1}-E_{0}\right| & \leq C \exp \left(-c(\log n)^{C_{0}}\right),
\end{aligned}
$$

and

$$
\log \left|f_{n}^{a}\left(x_{1}, E_{1}\right)\right| \geq n L_{n}\left(E_{1}\right)-(\log n)^{2 C_{0}} .
$$

Let $R=n^{-2 C_{3}}$, where $C_{3}$ is the constant $C_{1}$ from Corollary 3.17, and let $v_{x, E}(r)=$ $\#\left\{E: f_{n}^{a}\left(x, E^{\prime}\right)=0,\left|E^{\prime}-E\right| \leq r\right\}$. Using Jensen's formula we have that

$$
\begin{aligned}
v_{x_{1}, E_{1}}(3 R) & \leq C \int_{0}^{4 R} \frac{v_{x_{1}, E_{1}}(t)}{t} d t \\
& =\frac{1}{2 \pi} \int_{0}^{2 \pi} \log \left|f_{n}^{a}\left(x_{1}, E_{1}+4 R e^{i \theta}\right)\right| d \theta-\log \left|f_{n}^{a}\left(x_{1}, E_{1}\right)\right| .
\end{aligned}
$$


By Proposition 3.14 we have

$$
\log \left|f_{n}^{a}\left(x_{1}, E\right)\right| \leq n L_{n}^{a}(E)+C(\log n)^{p}
$$

for $E \in \mathscr{D}$. Using this, together with (4.19) and (4.20) yields

$$
v_{x_{1}, E_{1}}(3 R) \leq C\left(\sup _{\left|E-E_{1}\right|=4 R}\left(n\left(L_{n}^{a}(E)-L_{n}^{a}\left(E_{1}\right)\right)\right)+(\log n)^{2 C_{0}}\right) .
$$

For $E$ such that $\left|E-E_{0}\right| \leq R$ we have that $\left|E-E_{1}\right| \leq n^{-C_{3}}$ and hence by Corollary 3.17 we have that $\left|n\left(L_{n}^{a}(E)-L_{n}^{a}\left(E_{1}\right)\right)\right| \leq n^{-C}$. We can now conclude that

$$
v_{x_{1}, E_{0}}(2 R) \leq v_{x_{1}, E_{1}}(3 R) \leq C(\log n)^{2 C_{0}} .
$$

Using the Mean Value Theorem we can conclude that

$$
\left\|H^{(n)}\left(x_{0}\right)-H^{(n)}\left(x_{1}\right)\right\| \leq C n\left|x_{0}-x_{1}\right| \leq C \exp \left(-c(\log n)^{C_{0}}\right) .
$$

Let $E_{j}^{(n)}(x), j=1, \ldots, n$ be the eigenvalues of $H^{(n)}(x)$ ordered increasingly. Since $H^{(n)}\left(x_{0}\right)$ and $H^{(n)}\left(x_{1}\right)$ are Hermitian it follows that

$$
\left|E_{j}^{(n)}\left(x_{0}\right)-E_{j}^{(n)}\left(x_{1}\right)\right| \leq C \exp \left(-c(\log n)^{C_{0}}\right)
$$

This implies that $v_{x_{0}, E_{0}}(R) \leq v_{x_{1}, E_{0}}(2 R)$ and now the first estimate follows from eq. (4.21).

The second estimate follows in a similar way. From Proposition 4.10 it follows that there exists $x_{1}$ such that $\left|x_{1}-x_{0}\right| \leq C \exp \left(-c(\log n)^{C_{0}}\right)$ and

$$
\log \left|f_{n}^{a}\left(x_{1}, E_{0}\right)\right| \geq n L_{n}\left(E_{0}\right)-(\log n)^{2 C_{0}} .
$$

Let $v_{x}(r)=\#\left\{z \in \mathbb{C}: f_{n}^{a}\left(z, E_{0}\right)=0,|z-x|<r\right\}$. Using Jensen's formula, (4.22) and Proposition 3.14, as before, yields

$$
\begin{aligned}
v_{x_{0}}\left(n^{-1}\right) & \leq v_{x_{1}}\left(2 n^{-1}\right) \\
& \leq C\left(\sup _{r \in\left(1-3 n^{-1}, 1+3 n^{-1}\right)}\left(n\left(L_{n}^{a}\left(r, E_{0}\right)-L_{n}^{a}\left(1, E_{0}\right)\right)\right)+(\log n)^{2 C_{0}}\right) \\
& \leq C^{\prime}(\log n)^{2 C_{0}} .
\end{aligned}
$$

For the last inequality we used Corollary 3.13. This concludes the proof. 


\section{References}

[1] J. Bourgain, M. Goldstein, and M. Schlag, Anderson localization for Schrödinger operators on $\mathbb{Z}$ with potentials given by the skew-shift. Comm. Math. Phys. 220 (2001), 583-631. MR 1843776 Zbl 0994.82044 link.springer.com/article/10.1007\%2FPL00005570

[2] J. Bourgain and S. Jitomirskaya, Continuity of the Lyapunov exponent for quasiperiodic operators with analytic potential. Dedicated to David Ruelle and Yasha Sinai on the occasion of their $65^{\text {th }}$ birthdays. J. Statist. Phys. 108 (2002), 1203-1218. MR 1933451 Zbl 1039.81019

[3] J. Bourgain, Green's function estimates for lattice Schrödinger operators and applications. Annals of Mathematics Studies 158. Princeton University Press, Princeton, NJ, 2005. MR 2100420 Zbl 1137.35001

[4] M. Goldstein and W. Schlag, Hölder continuity of the integrated density of states for quasi-periodic Schrödinger equations and averages of shifts of subharmonic functions. Ann. of Math. (2) 154 (2001), 155-203. MR 1847592 Zbl 0990.39014

[5] M. Goldstein and W. Schlag, Fine properties of the integrated density of states and a quantitative separation property of the Dirichlet eigenvalues. Geom. Funct. Anal. 18 (2008), 755-869. MR 2438997 Zbl 1171.82011

[6] S. Jitomirskaya, D. A. Koslover, and M. S. Schulteis, Localization for a family of onedimensional quasiperiodic operators of magnetic origin. Ann. Henri Poincaré 6 (2005), 103-124. MR 2121278 Zbl 1062.81029

[7] S. Jitomirskaya, D. A. Koslover, and M. S. Schulteis, Continuity of the Lyapunov exponent for analytic quasiperiodic cocycles. Ergodic Theory Dynam. Systems 29 (2009), 1881-1905. MR 2563096 Zbl 1209.37016

[8] S. Jitomirskaya and C. A. Marx, Continuity of the Lyapunov exponent for analytic quasiperiodic cocycles with singularities. J. Fixed Point Theory Appl. 10 (2011), 129-146. MR $2825743 \mathrm{Zbl} 1236.37008$

[9] S. Jitomirskaya and C. A. Marx, Analytic quasi-perodic cocycles with singularities and the Lyapunov exponent of extended Harper's mode. Comm. Math. Phys. 316 (2012), 237-267. MR 2989459

[10] K. Tao, Hölder continuity of Lyapunov exponent for quasi-periodic Jacobi operators. Preprint 2011. arXiv:1108.3747

[11] G. Teschl, Jacobi operators and completely integrable nonlinear lattice. Mathematical Surveys and Monographs 72. Amer. Math. Soc., Providence, RI, 2000. MR 1711536 Zbl 1056.39029

Received February 13, 2012; revised May 14, 2012

Ilia Binder, Department of Mathematics, University of Toronto, Toronto, ON, M5S 2E4, Canada

E-mail: ilia@math.utoronto.ca

Mircea Voda, Department of Mathematics, University of Toronto, Toronto, ON, M5S 2E4,

Canada

E-mail:mvoda@math.utoronto.ca 